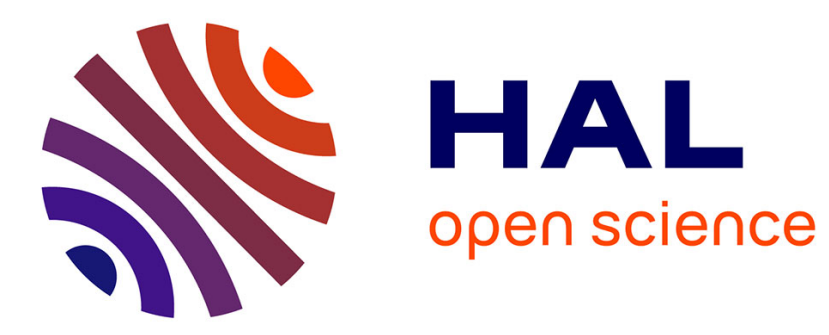

\title{
A robust finite element redistribution approach for elastodynamic contact problems
}

Farshid Dabaghi, Adrien Petrov, Jérôme Pousin, Yves Renard

\section{To cite this version:}

Farshid Dabaghi, Adrien Petrov, Jérôme Pousin, Yves Renard. A robust finite element redistribution approach for elastodynamic contact problems. Applied Numerical Mathematics, 2016, 103, pp.48-71. 10.1016/j.apnum.2015.12.004 . hal-00917450v2

\section{HAL Id: hal-00917450 \\ https://hal.science/hal-00917450v2}

Submitted on 15 Nov 2017

HAL is a multi-disciplinary open access archive for the deposit and dissemination of scientific research documents, whether they are published or not. The documents may come from teaching and research institutions in France or abroad, or from public or private research centers.
L'archive ouverte pluridisciplinaire HAL, est destinée au dépôt et à la diffusion de documents scientifiques de niveau recherche, publiés ou non, émanant des établissements d'enseignement et de recherche français ou étrangers, des laboratoires publics ou privés. 


\title{
A robust finite element redistribution approach for elastodynamic contact problems
}

\author{
Farshid Dabaghi ${ }^{\mathrm{a}}$, Adrien Petrov $^{\mathrm{a}}$, Jérôme Pousin ${ }^{\mathrm{a}}$, Yves Renard ${ }^{\mathrm{a}}$ \\ ${ }^{a}$ Université de Lyon, CNRS, INSA-Lyon, Institut Camille Jordan UMR 5208, 20 \\ Avenue A. Einstein, F-69621 Villeurbanne, France
}

\begin{abstract}
This paper deals with a one-dimensional elastodynamic contact problem and aims to highlight some new numerical results. A new proof of existence and uniqueness results is proposed. More precisely, the problem is reformulated as a differential inclusion problem, the existence result follows from some a priori estimates obtained for the regularized problem while the uniqueness result comes from a monotonicity argument. An approximation of this evolutionary problem combining the finite element method as well as the mass redistribution method which consists on a redistribution of the body mass such that there is no inertia at the contact node, is introduced. Then two benchmark problems, one being new with convenient regularity properties, together with their analytical solutions are presented and some possible discretizations using different time-integration schemes are described. Finally, numerical experiments are reported and analyzed.
\end{abstract}

Keywords: Numerical solution, mass redistribution method, variational inequality, unilateral contact, energy conservation.

2008 MSC: 35L05, 35L85, 49A29, 65N10, 65N30, 74M15.

\section{Introduction}

This paper aims to give some new numerical results for the dynamical evolution with impact of a linearly elastic body and a rigid obstacle by using

Email addresses: farshid.dabaghi@insa-lyon.fr (Farshid Dabaghi), apetrov@math.univ-lyon1.fr (Adrien Petrov), jerome.pousin@insa-lyon.fr (Jérôme Pousin), Yves.Renard@insa-lyon.fr (Yves Renard) 
the mass redistribution method introduced in [15]. The situations involving contact abound in industrial or biomedical problems. For this reason a considerable engineering and mathematical literature has been devoted to contact problems. Usually, with the hypotheses of small deformations, the contact phenomena is modelled by using the so-called Signorini's boundary conditions in displacement, which are based on a linearization of the physically meaningful non penetrability of masses. The elastodynamic contact problems are typically stated as hyperbolic type variational inequalities in Sobolev spaces, and a few existence of solutions results has been established. The reader is referred to $[30,21,16]$ and also the comprehensive monograph [9]. The uniqueness result was successfully investigated for a wave equation in a half-space with a unilateral contact at the boundary in [21]. In particular, the uniqueness still remains an open problem in other frameworks. The fundamental mathematical difficulties are related to the intrinsic nonsmoothness of the problem coming from the Signorini boundary conditions. Finally, notice that the existence of a strong solution for viscoelastodynamic problems with unilateral constraints is proved in [26].

On the other hand, another challenging task consists to elaborate efficient numerical methods for solving dynamical contact problems. Newmark's method is one of the most popular numerical solvers which is usually combined with finite elements methods. Unfortunately, for almost all values of the parameters of this method, unphysical energy blow-ups occur during the time integration as well as numerical instabilities at the dynamical contact boundaries. To overcome these difficulties, some numerical methods based on the Newmark scheme for solving impact problems and which are energy dissipative have been introduced in [3]. Although stable, those methods lead to an important energy loss at impact which do not vanish when the time step decreases. Laursen et al. in $[20,19,4,18]$ have designed time integration schemes of Newmark type which are energy conserving. However, these schemes are unable to prevent some spurious oscillations of the displacement and of the contact stress on the contact boundary. These unphysical oscillations are avoided by the numerical methods developed in [6] (see also the reference therein), but these methods are still energy dissipative. Another approach consisting to remove the mass at the contact nodes is investigated in [15]. This mass redistribution method prevents the oscillations at the contact boundaries mentioned above, and it can be proven that the solution is unique and energy conserving, see [5]. Furthermore, some different variants has been proposed in [11] with an estimate of the space approximation for 
linear elasticity. It has also been extended for thin structures in $[28,27,31]$ considering different discretizations for the displacement and the velocity.

The paper is organised as follows. In Section 2, the mathematical models are presented and a new proof of existence and uniqueness results is proposed. A space semi-discretization based on a redistribution of mass is introduced in Section 3 and some time integration method are advertised. In Sections 4 and 5, two elastodynamic contact benchmark problems with their analytical solutions are exhibited. Note that an analytical solution associated to the first benchmark problem is expressed by using Taylor expansion (see $[32,7])$ and it is compared to the approximated solution obtained thanks to Crank-Nicolson method. However, the numerical experiments highlight that the comparison between the analytical and approximated solutions do not converge. Therefore a new analytical solution displayed for the second benchmark is exposed in Section 5 and some numerical experiments problem are reported like convergence rates for the displacement, Lagrange multiplier and energy evolution showing the efficiency of mass redistribution method.

\section{Description of the models}

We consider a model for an elastic bar of length $L$ vibrating longitudinally. One end of this bar is free to move, as long as it does not hit a material obstacle, while the other end can be clamped or free to move. The obstacle constrains the displacement of the extremity to be non negative. The material of the bar is supposed to be homogeneous and the theory of small deformations is considered. Let us denote by $x$ the spatial coordinate along the bar, with the origin at the material obstacle, let $u(x, t)$ be the displacement at time $t \in[0, T], T>0$ of the material point of spatial coordinate $x \in[0, L]$. Let $f(x, t)$ denotes a density of external forces, depending on time and space. In the case where the bar is clamped at one end (see Figure 5), the mathematical problem is formulated as follows:

$$
u_{t t}(x, t)-u_{x x}(x, t)=f(x, t), \quad(x, t) \in(0, L) \times(0, T),
$$

with Cauchy initial data

$$
u(x, 0)=u^{0}(x) \quad \text { and } \quad u_{t}(x, 0)=v^{0}(x), \quad x \in(0, L),
$$

and Signorini and Dirichlet boundary conditions at $x=0$ and $x=L$, respectively,

$$
0 \leq u(0, t) \perp u_{x}(0, t) \leq 0 \quad \text { and } \quad u(L, t)=0, \quad t \in[0, T]
$$


While in the case where the bar is free to move at one end (see Figure 1), the motion is governed by the momentum equilibrium equation (1) together with Cauchy initial data (2) and Signorini and Neumann boundary conditions at $x=0$ and $x=L$, respectively,

$$
0 \leq u(0, t) \perp u_{x}(0, t) \leq 0 \quad \text { and } \quad u_{x}(L, t)=0, \quad t \in[0, T] .
$$

Here $u_{t} \stackrel{\text { def }}{=} \frac{\partial u}{\partial t}$ and $u_{x} \stackrel{\text { def }}{=} \frac{\partial u}{\partial x}$. The orthogonality has the natural meaning; namely if we have enough regularity, it means that at the boundary $x=0$ the product $u(0, \cdot) u_{x}(0, \cdot)$ vanishes almost everywhere in time. If it is not the case, the above inequality is integrated on an appropriate set of test functions, leading to a weak formulation for the unilateral condition. Observe that from mathematical viewpoint, Signorini's condition means that when the bar touches the obstacle at $x=0$, its reaction can be only upwards, so that $u_{x}(0, \cdot) \leq 0$ on the set $\{t: u(0, \cdot)=0\}$. While in the case where the bar does not touch the obstacle, its end is free to move. More precisely, we have $u_{x}(0, \cdot)=0$ on the set $\{t: u(0, \cdot)>0\}$. We suppose that the initial displacement $u^{0}$ belongs to the $\mathrm{H}^{1}(0, L)$ and satisfies the compatibility conditions, i.e. $u^{0}(L)=0$ and $u^{0}(0) \geq 0$, the initial velocity $v^{0}$ belongs to $\mathrm{L}^{2}(0, L)$ and the density of external forces $f$ belongs to $\mathrm{L}^{2}\left(0, T ; \mathrm{L}^{2}(0, L)\right)$.

We describe now the weak formulations of both the two problems. For that purpose, it is convenient to introduce the following notations: $\mathrm{V}^{\text {Dir }} \stackrel{\text { def }}{=}$ $\left\{u \in \mathrm{H}^{1}(0, L): u(L)=0\right\}, \mathrm{V}^{\mathrm{Neu}} \stackrel{\text { def }}{=} \mathrm{H}^{1}(0, L)$ and $\mathrm{H} \stackrel{\text { def }}{=} \mathrm{L}^{2}(0, L)$. We denote by $\mathcal{K}$ the following convex set:

$$
\mathcal{K} \stackrel{\text { def }}{=}\left\{u \in \mathcal{H}_{2}: u(\cdot, t) \in \mathrm{K} \text { for almost every } t\right\},
$$

where $\mathcal{H}_{2} \stackrel{\text { def }}{=}\left\{u \in \mathrm{L}^{2}(0, T ; \mathrm{V}): u_{t} \in \mathrm{L}^{2}(0, T ; \mathrm{H})\right\}, \mathrm{K} \stackrel{\text { def }}{=}\{u \in \mathrm{V}: u(0) \geq 0\}$ and $\mathrm{V}=\mathrm{V}^{\text {Dir }}$ or $\mathrm{V}=\mathrm{V}^{\mathrm{Neu}}$ depending on the conditions taken in $x=L$. Therefore the weak formulations associated to (1)-(3) and to (1)-(2) together with (4) are obtained by multiplying (1) by $v-u$ and by integrating formally this result over $Q_{T} \stackrel{\text { def }}{=}(0, L) \times(0, T)$ to get

$$
\left\{\begin{array}{l}
\text { find } u \in \mathcal{K} \text { such that } \\
-\int_{0}^{L} v^{0}\left(v(\cdot, 0)-u^{0}\right) \mathrm{d} x-\int_{Q_{T}} u_{t}\left(v_{t}-u_{t}\right) \mathrm{d} x \mathrm{~d} t \\
+\int_{Q_{T}} u_{x}\left(v_{x}-u_{x}\right) \mathrm{d} x \mathrm{~d} t \geq \int_{Q_{T}} f(v-u) \mathrm{d} x \mathrm{~d} t \\
\text { for all } v \in \mathbb{K} \text { for which there exists } \zeta>0 \text { with } v=u \text { for } t \geq T-\zeta .
\end{array}\right.
$$


Notice that the sole difference of the weak formulation associated to (1)-(3) and to (1)-(2), (4) comes from the definition of the convex set $\mathcal{K}$.

It should also be noted that existence and uniqueness results are obtained for a similar situation of a vibrating string with concave obstacle in one dimensional space in [30] and also for a wave equations with unilateral constraint at the boundary in a half space of $\mathbb{R}^{N}$ in [21]. An existence result for a wave equation in a $\mathrm{C}^{2}$-regular bounded domain constrained by an obstacle at the boundary in $\mathbb{R}^{N}$ for $N \geq 2$ is proven in [16].

Theorem 2.1 (Existence and uniqueness results). Assume that $u^{0} \in \mathrm{V}$, $v^{0} \in \mathrm{H}$ and $f \in \mathrm{L}^{2}(0, T ; \mathrm{H})$. Then Problem (1)-(3) admits a unique solution $u \in \mathrm{L}^{\infty}(0, T ; \mathrm{V}) \cap \mathrm{W}^{1, \infty}(0, T ; \mathrm{H})$.

Proof. The first step consists to introduce an auxiliary problem. Namely, let $\bar{u}$ be the solution to Problem (1) with initial data (2) and boundary conditions (3) where Signorini's boundary condition is replaced by Dirichlet's one, i.e. $\bar{u}(0, t)=0, t \in[0, T]$. Note that the auxiliary problem possesses a unique solution $\bar{u}$ belonging to $\mathrm{C}^{0}\left([0, T] ; \mathrm{H}^{2}(0, L) \cap \mathrm{H}_{0}^{1}(0, L)\right) \cap \mathrm{C}^{1}\left([0, T] ; \mathrm{H}_{0}^{1}(0, L)\right) \cap$ $\mathrm{C}^{2}([0, T] ; \mathrm{H})($ see $[22,2])$. Then we denote by $v \stackrel{\text { def }}{=} u-\bar{u}$ a solution of

$$
v_{t t}(x, t)-v_{x x}(x, t)=0, \quad(x, t) \in(0, L) \times(0, T),
$$

with Cauchy initial data

$$
v(x, 0)=0 \quad \text { and } \quad v_{t}(x, 0)=0, \quad x \in(0, L),
$$

and Signorini and Dirichlet boundary conditions at $x=0$ and $x=L$, respectively,

$$
0 \leq v(0, t) \perp v_{x}(0, t)+\bar{u}_{x}(0, t) \leq 0 \quad \text { and } \quad v(L, t)=0, \quad t \in[0, T] .
$$

The second step consists to rewrite (6)-(8) as a differential inclusion problem by using the characteristic method. To this aim, it is convenient to introduce the characteristic coordinates $\xi \stackrel{\text { def }}{=} x+t$ and $\eta \stackrel{\text { def }}{=} x-t$. Therefore the chain rule gives $v_{x x}=v_{\xi \xi}+2 v_{\xi \eta}+v_{\eta \eta}$ and $v_{t t}=v_{\xi \xi}-2 v_{\xi \eta}+v_{\eta \eta}$. According to (1), $v_{\xi \eta}$ vanishes and it follows that $v(\xi, \eta)=\phi(\xi)+\psi(\eta)$, where $\phi$ and $\psi$ are two differentiable functions such that $v(x, t)=\phi(x+t)+\psi(x-t)$. In particular, taking $t=0$ and using the Cauchy initial data, we get $\phi(x)+\psi(x)=0$ and $\phi^{\prime}(x)-\psi^{\prime}(x)=0$, which by integration implies that there exist two constants 
$C^{\phi}$ and $C^{\psi}$ such that $\phi(\xi)=C^{\phi}$ and $\psi(\eta)=C^{\psi}$ for all $\xi$ and $\eta$ belonging to $[0, L]$. It comes that the boundary conditions (3) can be rewritten as follows:

$0 \leq \phi(t)+\psi(-t) \perp \phi^{\prime}(t)+\psi^{\prime}(-t)+g(t) \leq 0 \quad$ and $\quad \phi(L+t)+\psi(L-t)=0$

for all $t$ belonging to $[0, T]$. Thanks to the above identity, we may extend $\phi(t)$ for all $t \in[L, 2 L]$, i.e. we have

$$
\phi(L+t)=-\psi(L-t)
$$

for all $t$ belonging to $[0, L]$. If we choose $t^{\prime}=L+t$, we get $\phi\left(t^{\prime}\right)=-\psi\left(2 L-t^{\prime}\right)$. We already have the solution for $\psi(t)$ with $0 \leq t \leq L$ and if $L \leq t^{\prime} \leq 2 L$, we can obtain $\phi\left(t^{\prime}\right)$ by observing that $0 \leq 2 L-t^{\prime} \leq L$ and by using $\psi(2 L-t)$, it comes that $\phi(t)=C^{\psi}$ for all $t$ belonging to $[L, 2 L]$.

Let the indicator function, namely $\chi_{(-\infty, 0]}(x)$ is equal to 0 if $x \in(-\infty, 0]$ and $+\infty$ otherwise. Obviously, $\chi_{(-\infty, 0]}$ is a lower semi-continuous and convex function, for further details the reader is referred to [1]. Its subdifferential is a multivalued function $J_{N}: \mathbb{R} \rightarrow \mathcal{P}(\overline{\mathbb{R}})$ defined by

$$
J_{N}[x] \stackrel{\text { def }}{=}\left\{\begin{array}{lll}
\{0\} & \text { if } & x<0 \\
{[0,+\infty)} & \text { if } & x=0 \\
\emptyset & \text { if } & x>0
\end{array}\right.
$$

where $\mathcal{P}(\overline{\mathbb{R}})$ is the set of all subsets of $\overline{\mathbb{R}}$. Then, the inequalities in (9) can be rewritten as follows

$$
\phi^{\prime}(t)+\psi^{\prime}(-t)+g(t) \in-J_{N}[-\phi(t)-\psi(-t)] .
$$

Note that at this stage, $\psi(\eta), \eta \in[-L, L]$, is the unique unknown of (10). Let us define now

$$
w(t) \stackrel{\text { def }}{=}-\phi(t)-\psi(-t) .
$$

We insert (11) into (10) to get

$$
w^{\prime}(t) \in-J_{N}[w(t)]-g(t) .
$$

Finally, we find the following Cauchy problem

$$
\begin{aligned}
& w^{\prime}(t) \in-J_{N}[w(t)]-g(t) \quad \text { a.e. } \quad t \in(0, L), \\
& w(0)=0 .
\end{aligned}
$$


Then we look for a solution of the following problem:

$$
\begin{aligned}
& w^{\prime}(t)+h(t)=-g(t) \quad \text { a.e. } \quad t \in(0, L), \\
& h(t) \in J_{N}[w(t)] \quad \text { a.e. } \quad t \in(0, L), \\
& w(0)=0 .
\end{aligned}
$$

We observe that (13) is equivalent to (9), the verification is left to the reader. We regularize the problem (13), we construct a solution of the regularized problem, and we show the existence of a solution by passing to the limit with respect to regularity parameter.

We regularize the problem (13) by using Yosida's regularization. More precisely, we introduce first a sequence $\left\{g_{\varepsilon}\right\}_{\varepsilon>0}$ such that $g_{\varepsilon}$ belongs to $\mathrm{C}^{0}([0, L])$ for all $\varepsilon>0$ and

$$
g_{\varepsilon} \rightarrow g \text { in } \mathrm{H} \quad \text { as } \varepsilon \searrow 0 .
$$

We may approximate (13) by the following problem:

$$
\begin{aligned}
& w_{\varepsilon}^{\prime}(t)+J_{N, \varepsilon}\left[w_{\varepsilon}(t)\right]=-g_{\varepsilon}(t) \quad \text { a.e. } \quad t \in(0, L), \\
& w_{\varepsilon}(0)=0 .
\end{aligned}
$$

where $J_{N, \varepsilon} \stackrel{\text { def }}{=} \frac{1}{\varepsilon}\left(1-\mathcal{J}_{\varepsilon}\right)$ denotes the Yosida approximation with the resolvant defined by $\mathcal{J}_{\varepsilon} \stackrel{\text { def }}{=}\left(1+\varepsilon J_{N}\right)^{-1}$. Note that $J_{N, \varepsilon}$ is a monotone and Lipschitz continuous mapping defined on all $\mathrm{H}$. Clearly the contraction principle step by step in time allows us to prove that (13) possesses a unique solution $w_{\varepsilon}$ belonging to $\mathrm{C}^{1}([0, L])$.

We establish now some a priori estimates, which later will enable to infer the existence of a weak solution. To this aim, we multiply (15a) by $w_{\varepsilon}^{\prime}(t)$, and we integrate this expression over $(0, L)$ to get

$$
\int_{0}^{L}\left|w_{\varepsilon}^{\prime}(t)\right|^{2} \mathrm{~d} t+\int_{0}^{L} J_{N, \varepsilon}\left[w_{\varepsilon}(t)\right] w_{\varepsilon}^{\prime}(t) \mathrm{d} t=-\int_{0}^{L} g_{\varepsilon}(t) w_{\varepsilon}^{\prime}(t) \mathrm{d} t .
$$

Let us define now

$$
I_{(-\infty, 0]}^{\varepsilon}(w) \stackrel{\text { def }}{=} \min _{z \in \mathrm{H}}\left\{\frac{1}{2 \varepsilon}|w-z|^{2}+I_{(-\infty, 0]}(z)\right\}
$$

for all $\varepsilon>0$ and $w \in \mathrm{H}$. Since $J_{N}$ is the subdifferential of a proper, convex and lower semicontinuous function $I_{(-\infty, 0]}$, it follows that $I_{(-\infty, 0]}^{\varepsilon}$ is convex, 
Fréchet differentiable in $\mathrm{H}$ and its subdifferential $\partial I_{(-\infty, 0]}^{\varepsilon}$ coincides with $J_{N, \varepsilon}$. Furthermore we have

$$
\forall z \in \mathrm{H}: I_{(-\infty, 0]}^{\varepsilon}(z) \nearrow I_{(-\infty, 0]}(z) \text { as } \varepsilon \searrow 0 .
$$

Then we may deduce from (16) that

$$
\frac{1}{2}\left\|w_{\varepsilon}^{\prime}\right\|_{\mathrm{H}}^{2}+I_{(-\infty, 0]}^{\varepsilon}\left(w_{\varepsilon}(L)\right) \leq \frac{1}{2}\left\|g_{\varepsilon}\right\|_{\mathrm{H}}^{2} .
$$

We observe that by definition $I_{(-\infty, 0]}^{\varepsilon}\left(w_{\varepsilon}(L)\right) \geq 0$ which follows from (17) that there exists $C_{1}>0$, independent of $\varepsilon>0$, such that

$$
\left\|w_{\varepsilon}^{\prime}\right\|_{\mathrm{H}} \leq C_{1} .
$$

Furthermore we have

$$
\left|w_{\varepsilon}(\tau)\right|^{2} \leq 2 L\left\|w_{\varepsilon}^{\prime}\right\|_{\mathrm{H}}^{2}
$$

for all $\tau \in(0, L)$. Then we deduce from (17) and (19) that there exists $C_{2}>0$, independent of $\varepsilon>0$, such that

$$
\left|w_{\varepsilon}(\tau)\right|^{2}+\left\|w_{\varepsilon}^{\prime}\right\|_{\mathrm{H}}^{2} \leq C_{2}
$$

for all $\tau \in(0, L)$. Since $\mathcal{J}_{\varepsilon}$ is a contraction on all $\mathrm{H}$ (see [1]), it follows from (20) that there exists $C_{3}>0$, independent of $\varepsilon>0$, such that

$$
\left\|w_{\varepsilon}\right\|_{\mathrm{V}}+\left\|\mathcal{J}_{\varepsilon} w_{\varepsilon}\right\|_{\mathrm{V}} \leq C_{3} .
$$

We observe that (15a) implies that

$$
\left\|J_{N, \varepsilon}\left[w_{\varepsilon}\right]\right\|_{\mathrm{H}}^{2} \leq 2\left\|w_{\varepsilon}^{\prime}\right\|_{\mathrm{H}}^{2}+2\left\|g_{\varepsilon}\right\|_{\mathrm{H}}^{2} .
$$

The above a priori estimates allow us to infer that there exists $w$ and $h$ belonging to $\mathrm{V} \cap \mathrm{L}^{\infty}(0, L)$, respectively, and passing to subsequences, if necessary, we find

$$
\begin{aligned}
\mathcal{J}_{\varepsilon} w_{\varepsilon} \rightarrow w & \text { in } \quad \mathrm{L}^{\infty}(0, L) \quad \text { weak } \quad *, \\
J_{N, \varepsilon}\left[w_{\varepsilon}\right] \rightarrow h \quad \text { in } \quad \mathrm{H} \quad \text { weak, } &
\end{aligned}
$$

as $\varepsilon$ tends to 0 . Since $\mathrm{V} \hookrightarrow \mathrm{H}$ with compact embedding, it follows that

$$
\begin{aligned}
\mathcal{J}_{\varepsilon} w_{\varepsilon} \rightarrow w & \text { in } \mathrm{H}, \\
w_{\varepsilon} \rightarrow w & \text { in } \mathrm{H} \quad \text { and } \quad w_{\varepsilon} \rightarrow w \quad \text { in } \mathrm{V} \quad \text { weak, }
\end{aligned}
$$


as $\varepsilon$ tends to 0 . It remains to prove that $w$ and $h$ satisfy (13). We integrate (15a) over $(0, \tau)$, we get

$$
\int_{0}^{\tau}\left(w_{\varepsilon}^{\prime}(t)+J_{N, \varepsilon}\left[w_{\varepsilon}(t)\right]+g_{\varepsilon}(t)\right) \mathrm{d} t=0
$$

for all $\tau \in(0, L)$. Thanks to (23) and (24), we may pass to the limit in all the terms of (25), namely we find

$$
\int_{0}^{\tau}\left(w^{\prime}(t)+h(t)+g(t)\right) \mathrm{d} t=0
$$

for all $\tau \in(0, L)$, which is equivalent to (13a). Let us note that $J_{N, \varepsilon}\left[w_{\varepsilon}\right] \in$ $J_{N}\left[\mathcal{J}_{\varepsilon} w_{\varepsilon}\right]$, for some technical details, the reader is referred to [1]. Then (23b) and (24a) enable us to deduce that

$$
\limsup _{\varepsilon \searrow 0} \int_{0}^{L} J_{N, \varepsilon}\left[w_{\varepsilon}(t)\right] \mathcal{J}_{\varepsilon} w_{\varepsilon}(t) \mathrm{d} t \leq \int_{0}^{L} h(t) w(t) \mathrm{d} t,
$$

which implies that (13b) holds. Then we may conclude that there exists a solution $v$ to Problem (6)-(8).

The uniqueness result comes from the monotonicity of $J_{N}$, namely $J_{N}$ is the subdifferential of a convex, lower semi-continuous and proper function (see [1] for further details).

The existence and uniqueness results to Problem (1)-(2) together with (4) is established by using analogous approach developed above for Problem (1)-(3). The main difference comes from the choice of the auxiliary problem. Let $\bar{u}$ be the unique solution to Problem (1) with initial data (2) and boundary conditions (4) where the Signorini boundary conditions are replaced by Dirichlet boundary conditions, i.e. $\bar{u}(0, t)=0, t \in[0, T]$. Hence the rest of the proof is quite a routine, the verification is left to the reader.

Finally, we consider the following energy which is associated to (5):

$$
\mathcal{E}(t)=\frac{1}{2} \int_{0}^{L}\left(\left|u_{t}(x, t)\right|^{2}+\left|u_{x}(x, t)\right|^{2}\right) \mathrm{d} x .
$$

This energy is constant with respect to time $t$ when the density forces $f$ vanishes.

Note that formulations (5) and (13) are equivalent (in the sense that they have the same weak solutions), since the proof given in [5] can be straightforwardly adapted. 


\section{Discretization}

\subsection{Classical finite element discretization}

We introduce now a semi-discrete problem in space associated to (5). To this aim, we choose a set of parameters $h \stackrel{\text { def }}{=} \frac{L}{m}$ (mesh size) having in mind the limit $m \rightarrow+\infty$, where $m$ is an integer, and let $\mathrm{V}_{h}^{\text {Neu }} \stackrel{\text { def }}{=}\left\{v_{h} \in \mathrm{C}^{0}([0, L])\right.$ : $\left.\left.v_{h}\right|_{\left[a_{i}, a_{i+1}\right]} \in P_{1}, i=0, \ldots, m-1\right\}$ and $\mathrm{V}_{h}^{\text {Dir }} \stackrel{\text { def }}{=}\left\{v_{h} \in \mathrm{V}_{h}^{\text {Neu }}: v_{h}(L)=0\right\}$. Here, $a_{i} \stackrel{\text { def }}{=} i h, i=0,1, \ldots, m$ and $P_{1}$ is the space of polynomials of degree less than or equal to 1 . A classical basis of $V_{h}$ is given by the sequence of shape functions $\varphi_{i} \in \mathrm{V}_{h}$ for $i=0,1, \ldots, J$, defined by

$$
\varphi_{i}(x) \stackrel{\text { def }}{=} \begin{cases}1-\frac{\left|x-a_{i}\right|}{h} & \text { if } \quad x \in\left[a_{\max (i-1,0)}, a_{\min (i+1, m)}\right], \\ 0 & \text { otherwise }\end{cases}
$$

where $J=m-1$ in the case where $\mathrm{V}_{h}=\mathrm{V}_{h}^{\text {Dir }}$ and $J=m$ in the case where $\mathrm{V}_{h}=\mathrm{V}_{h}^{\text {Neu }}$. Observe that $\varphi_{i}\left(a_{j}\right)=\delta_{i j}, j=0,1, \ldots, m$ ( $\delta$ is Kronecker's symbol). We approximate the solution $u$ belonging to $\mathrm{V}$ to the weak formulation (5) by

$$
u_{h}(x, t)=\sum_{j=0}^{J} u_{j}(t) \varphi_{j}(x) .
$$

Consequently, we have $u_{i}=u_{h}\left(a_{i}\right), i=0,1, \ldots, J$. We shall consider two strategies to approximate the dynamic contact problem. The first one is a classical finite element semi-discretization which uses a multiplier. It reads as follows:

$$
\left\{\begin{array}{l}
\text { find } u_{h}:[0, T] \rightarrow \mathrm{V}_{h} \text { and } \lambda:[0, T] \rightarrow \mathbb{R} \text { such that for all } v_{h} \in \mathrm{V}_{h} \\
\int_{0}^{L} u_{h, t t} v_{h} \mathrm{~d} x+\int_{0}^{L} u_{h, x} v_{h, x} \mathrm{~d} x=-\lambda v_{h}(0)+\int_{0}^{L} f v_{h} \mathrm{~d} x \quad \text { a.e. } \quad t \in[0, T], \\
0 \leq u_{h}(0, \cdot) \perp \lambda \leq 0 \quad \text { a.e. } \quad t \in[0, T], \\
u_{h}(\cdot, 0)=u_{h}^{0} \quad \text { and } \quad u_{h, t}(\cdot, 0)=v_{h}^{0},
\end{array}\right.
$$

where $u_{h}^{0}$ and $v_{h}^{0}$ belong to $\mathrm{V}_{h}$ and $\lambda$ is the Lagrange multiplier being here the contact force. Furthermore, the approximated problem (27) can be rewritten 
in an algebraic formulation form as follows:

$$
\left\{\begin{array}{l}
\text { find } U_{h}:[0, T] \rightarrow \mathbb{R}^{n} \text { and } \lambda:[0, T] \rightarrow \mathbb{R} \text { such that } \\
M U_{h, t t}+S U_{h}=-\lambda e_{0}+F \quad \text { a.e. } \quad t \in[0, T] \\
0 \leq u_{0} \perp \lambda \leq 0 \quad \text { a.e. } \quad t \in[0, T] \\
U_{h}(0)=U_{h}^{0} \quad \text { and } \quad U_{h, t}(0)=V_{h}^{0},
\end{array}\right.
$$

where $M$ and $S$ denote the mass and stiffness matrices, respectively, and $U \stackrel{\text { def }}{=}$ $\left(u_{0}, \ldots, u_{n-1}\right)^{\top}, e_{0} \stackrel{\text { def }}{=}(1,0, \ldots, 0)^{\top}$ and $F \stackrel{\text { def }}{=}\left(\int_{0}^{L} f \varphi_{0} \mathrm{~d} x, \ldots, \int_{0}^{L} f \varphi_{n-1} \mathrm{~d} x\right)$.

\subsection{Finite element discretization with the mass redistribution method}

An alternative to the standard discretization is to consider a trivial mass redistribution method which consists to replace the mass matrix $M$ in (28) by a modified mass matrix $M^{\text {mod }}$ defined by

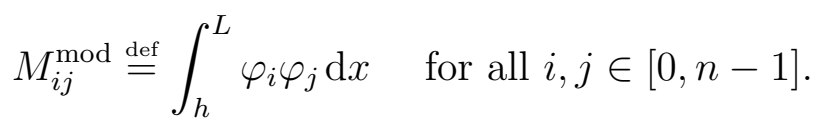

Let us observe that there are more sophisticated mass redistribution methods, see [12]. The modified mass matrix has the form

$$
M^{\mathrm{mod}} \stackrel{\text { def }}{=}\left(\begin{array}{cc}
0 & 0 \\
0 & \bar{M}
\end{array}\right)
$$

where $\bar{M}$ admits an unmodified part $\bar{M}_{i j}=M_{i+1, j+1}$, for all $i, j=1, \ldots, n-2$. As a consequence and in a certain sense, the node at the contact boundary evolves in a quasi-static way. It has been established in [5] in a slightly different context that the approximated solution using the mass redistribution method converges to the unique solution to (5).

Finally, note that the discrete energy associated to problem (28) is given by

$$
\mathcal{E}_{h}(t)=\frac{1}{2}\left(U_{h, t}^{\top} M U_{h, t}+U_{h}^{\top} S U_{h}-U_{h, t}^{\top} F\right)(t) .
$$

We introduce now the time discretization. In order to fix the notations, let the time interval $[0, T]$ be divided by $n+1$ discrete time-points such that $0=t_{0}<t_{1}<\ldots<t_{n}=T$. Furthermore the discrete quantities $U_{h}^{n}, U_{h, t}^{n}$, $U_{h, t t}^{n}$ and $\lambda^{n}$ are assumed to be given by algorithmic approximations of the displacement $U_{h}\left(t_{n}\right)$, the velocity $U_{h, t}\left(t_{n}\right)$ and the acceleration $U_{h, t t}\left(t_{n}\right)$ and 
the Lagrange multiplier $\lambda\left(t_{n}\right)$, respectively. Some time-stepping schemes allowing to obtain an approximated solution to Problem (1)-(4) are introduced below and their efficiency is discussed and analyzed in the next sections.

The most wide-spread time-stepping scheme for solving contact problems is the family of classical Newmark methods proposed by Newmark in [23]. The algorithms form a subset of the Hilber-Hughes-Taylor (HHT) family of temporal integrators, the reader is referred to [13] for further details. The underlying concept of the discretizations are Taylor expansions of displacements and velocities neglecting terms of higher order. For the contact problem introduced above, the discrete evolution is described by the finite difference equations:

$$
\begin{aligned}
& U_{h}^{n+1}=U_{h}^{n}+\Delta t U_{h, t}^{n}+\left(\frac{1}{2}-\beta\right) \Delta t^{2} U_{h, t t}^{n}+\beta \Delta t^{2} U_{h, t t}^{n+1}, \\
& U_{h, t}^{n+1}=U_{h, t}^{n}+(1-\gamma) \Delta t U_{h, t t}^{n}+\gamma \Delta t U_{h, t t}^{n+1}, \\
& M U_{h, t t}^{n+1}+S U_{h}^{n+1}=-\lambda^{n+1} e_{0}+F^{n+1}, \\
& 0 \leq u_{0}^{n+1} \perp \lambda^{n+1} \leq 0,
\end{aligned}
$$

where $\Delta t$ is a given time step and $(\beta, \gamma)$ are the algorithmic parameters. Furthermore $U_{h}^{0}, U_{h, t}^{0}$ and $\lambda^{0}$ are given and $U_{h, t t}^{0}$ is evaluated by using (30c). Notice that the Newmark family contains many well-known and widely-used algorithms which correspond to different choices of the algorithmic parameters $(\beta, \gamma)$ (see $[14,18])$. This method is unconditionally stable for linear elastodynamic problem for $\gamma \geq \frac{1}{2}$ and $\beta \geq \frac{1}{4}\left(\frac{1}{2}+\gamma\right)^{2}$ (see $[14,17]$ ). One of the most used method to study dynamic problems is the Crank-Nicolson method, also called trapezoidal or average acceleration method, which is obtained by setting the parameters $(\beta, \gamma)=\left(\frac{1}{4}, \frac{1}{2}\right)$. This method is second-order consistent and unconditionally stable in the unconstrained case. Furthermore, the total energy of the discrete evolution is preserved, for the purely elastic case, see [18]. That is the reason why the Crank-Nicolson method is commonly used in the community of computational mechanics. However, the situation should be examined in the case of contact constraints. Indeed the order of accuracy is degraded, for further details, the reader is referred to $[14,17,10]$. Let us define now the energy evolution by $\Delta \mathcal{E}_{h}^{n} \stackrel{\text { def }}{=} \mathcal{E}_{h}^{n+1}-\mathcal{E}_{h}^{n}$, where $\mathcal{E}_{h}^{n}$ is assumed to be given by an algorithmic approximation of the energy $\mathcal{E}_{h}\left(t_{n}\right)$. In particular, the energy evolution associated to $(30)$ for $(\beta, \gamma)=\left(\frac{1}{4}, \frac{1}{2}\right)$ is given by

$$
\Delta \mathcal{E}_{h}^{n}=-\left(V_{h}^{n}\right)^{\top} \Lambda_{\gamma}^{n} e_{0}
$$


where $V_{h}^{n} \stackrel{\text { def }}{=} U_{h}^{n+1}-U_{h}^{n}$ and $\Lambda_{\gamma}^{n} \stackrel{\text { def }}{=}(1-\gamma) \lambda^{n}+\gamma \lambda^{n+1}$. Let us observe that in the presence of permanent contact, the energy is strictly conserved, while the release of an existing contact decreases the energy and the detection of a new contact increases the energy. However, regain and loss of energy do not balance and there is no guarantee that the energy will stay bounded during the time integration.

Another approach to study elastodynamic contact problems consists to use the $\theta$-method. More precisely, the discrete evolution is described by the finite difference equations:

$$
\begin{aligned}
& U_{h}^{n+1}=U_{h}^{n}+\Delta t\left((1-\theta) U_{h, t}^{n}+\theta U_{h, t}^{n+1}\right), \\
& U_{h, t}^{n+1}=U_{h, t}^{n}+\Delta t\left((1-\theta) U_{h, t t}^{n}+\theta U_{h, t t}^{n+1}\right), \\
& M U_{h, t t}^{n+1}+S U_{h}^{n+1}=-\lambda^{n+1} e_{0}+F^{n+1}, \\
& 0 \leq u_{0}^{n+1} \perp \lambda^{n+1} \leq 0,
\end{aligned}
$$

with $\theta \in[0,1]$. Furthermore $U_{h}^{0}, U_{h, t}^{0}$ and $\lambda^{0}$ are given and $U_{h, t t}^{0}$ is evaluated by using (32c). The energy evolution associated to (32) is given by

$$
\Delta \mathcal{E}_{h}^{n}=\left(\frac{1}{2}-\theta\right)\left(V_{h, t}^{n}\right)^{\top} M V_{h, t}^{n}+\left(\frac{1}{2}-\theta\right)\left(V_{h}^{n}\right)^{\top} S V_{h}^{n}-\left(V_{h}^{n}\right)^{\top} \Lambda_{\theta}^{n} e_{0} .
$$

Notice that (32) with $\theta=0$ and $\theta=1$ are called the forward and backward Euler method, respectively, while with $\theta=\frac{1}{2}$, we obtain Crank-Nicolson's method.

Finally, we focus on the so-called Paoli-Schatzman method that consists to fix the contact constraint at an intermediate time step. Indeed the method proposed below is a slight modification of Paoli-Schatzman method (see [24, 25]) which takes into account the kernel of the modified mass matrix. A simple application of Paoli-Schatzman method based on Newmark scheme to Problem (28) with $\gamma=\frac{1}{2}$ leads to

$$
\left\{\begin{array}{l}
\text { find } U_{h}^{n+1}:[0, T] \rightarrow \mathbb{R}^{n} \text { and } \lambda^{n}:[0, T] \rightarrow \mathbb{R} \text { such that: } \\
\frac{M\left(U_{h}^{n+1}-2 U_{h}^{n}+U_{h}^{n-1}\right)}{\Delta t^{2}}+S\left(\beta U_{h}^{n+1}+(1-2 \beta) U_{h}^{n}+\beta U_{h}^{n-1}\right)= \\
-\lambda^{n} e_{0} \quad \text { for all } n \geq 2, \\
0 \leq u_{0}^{n, e}=\frac{u_{0}^{n+1}+e u_{0}^{n-1}}{1+e} \perp \lambda^{n} \leq 0, \\
U_{0} \text { and } U_{1} \text { given. }
\end{array}\right.
$$


Here $e$ belongs to $[0,1]$ and is aimed to be interpreted as a restitution coefficient. Note that $U_{h}^{0}$ and $U_{h}^{1}$ are given data and $U_{h}^{1}$ can be evaluated by a one step scheme. We may observe that taking $M=M^{\text {mod }}$ in (34), we are not able to resolve the problem on the kernel of $M^{\text {mod }}$. That is the reason why, $S U_{h}^{n-1}$ as well as $S U_{h}^{n}$ are projected on the orthogonal of the kernel of $M$. The energy evolution associated to (34) is given for $\beta=\frac{1}{4}$ by

$$
\Delta \mathcal{E}_{h}^{n}=\frac{1+e}{2} \lambda^{n} u_{0}^{n-1}
$$

Notice that the numerical simulations were performed by employing the finite element library Getfem ++ (see [29]). For the reader convenience, the energy evolutions (31), (33) and (35) are justified in the Appendix.

\section{The wave equation with Signorini and Neumann boundary con- ditions}

\subsection{Analytical solution}

We consider in this section the analytical solution proposed in [32]. It is the solution to the equation of motion (1) together with Cauchy initial data (2) and boundary conditions (4) in the case where the density of external forces $f(x, t)=-\left(g+u_{x}(0, t)\right)$ with $g>0$ being the gravity acceleration. Let us describe the solution of this problem (see Figure 1). Before the impact, the bar is undeformed and its initial velocity $v^{0}$ vanishes. The bar reaches the rigid obstacle at time $t_{1}=\sqrt{\frac{2 u^{0}}{g}}$ with the velocity $\sqrt{2 u^{0} g}$. After the impact, the bar stays in contact as long as the shock wave travels from bottom to the top of the bar and vice versa and then it takes off. Since the velocity of the shock wave $c$ is assumed to be equal to 1, the bar stays in contact with the rigid obstacle a time $L$. Finally, notice that impacts occur at $t_{4 k+1}=3 L+16 k L, t_{4 k+2}=t_{4 k+1}+2 L, t_{4 k+3}=t_{4 k+1}+8 L$ and $t_{4 k+4}=t_{4 k+1}+10 L$. We may deduce that the analytical solution is given by

$$
u(x, t)=\left\{\begin{array}{lll}
u^{0}-\frac{1}{2} g t^{2} & \text { if } \quad t \leq t_{1}, \\
h_{1}\left(x, t-t_{4 k+1}\right) & \text { if } \quad t_{4 k+1}<t \leq t_{4 k+2}, \\
h_{2}\left(x, t-t_{4 k+2}\right) & \text { if } \quad t_{4 k+2}<t \leq t_{4 k+3}, \\
h_{1}\left(x, t_{4 k+4}-t\right) & \text { if } \quad t_{4 k+3}<t \leq t_{4 k+4}, \\
u^{0}-\frac{1}{2} g\left(t-t_{4 k+4}-\sqrt{\frac{2 u^{0}}{g}}\right)^{2} & \text { if } \quad t_{4 k+4}<t \leq t_{4(k+1)+1} .
\end{array}\right.
$$




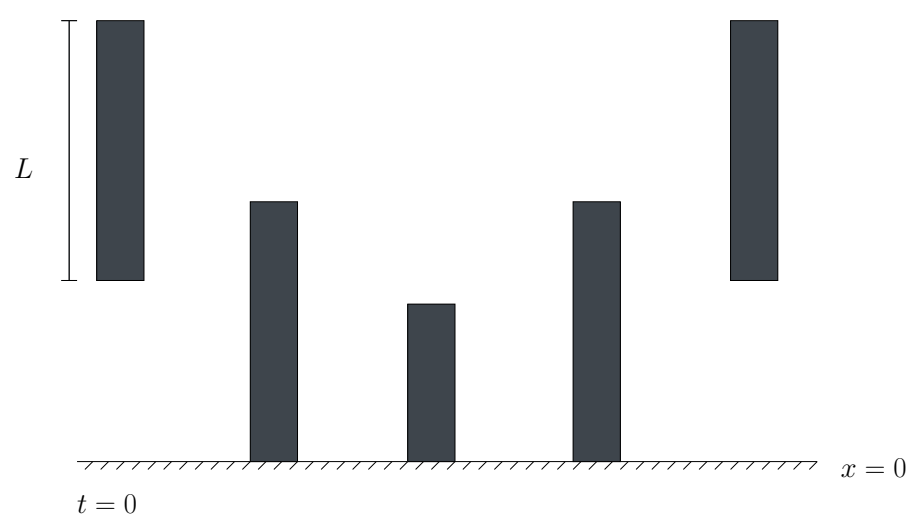

Figure 1: An elastic bar with both ends free to move

where we have used the following notations

$$
\begin{aligned}
& h_{1}(x, t) \stackrel{\text { def }}{=}-\sqrt{\frac{2 u^{0}}{g}} \min (x, L-|t-L|)+\sum_{n=1}^{+\infty} \frac{2 g}{L \nu_{n}^{3}}\left(\cos \left(\nu_{n} t\right)-1\right) \sin \left(\nu_{n} x\right), \\
& h_{2}(x, t) \stackrel{\text { def }}{=} u^{0}-\frac{1}{2} g\left(t-\sqrt{\frac{2 u^{0}}{g}}\right)^{2}-\frac{2 g L^{2}}{3}+\sum_{n=1}^{+\infty} \frac{4 g}{\lambda_{n}^{2}} \cos \left(\lambda_{n} t\right) \cos \left(\lambda_{n} x\right),
\end{aligned}
$$

with $\nu_{n} \stackrel{\text { def }}{=}\left(n-\frac{1}{2}\right) \frac{\pi}{L}$ and $\lambda_{n} \stackrel{\text { def }}{=} n \frac{\pi}{L}$.

\subsection{Numerical simulations}

The parameters used in the numerical simulations are $L=1, g=0.11$, $u^{0}(x)=0.5, v^{0}(x)=0$ and $u_{x}(L, \cdot)=0$. We evaluate the approximated solution $\left(U_{h}^{n}, \lambda^{n}\right)$ to Problem (1)-(3) by using Crank-Nicolson method that consists to choose $(\beta, \gamma)=\left(\frac{1}{4}, \frac{1}{2}\right)$ in (30) for the standard and modified mass matrices. Then we compare the solutions obtained by employing CrankNicolson method to the analytical one $(u, \lambda)$ in different points of the bar (see Figure 2). In the case where the constraint is active, the numerical experiments highlighted some spurious oscillations occurring for the approximated solution $\left(U_{h}^{n}, \lambda^{n}\right)$ obtained by solving (30) with the standard mass matrix $M$ (see Figure 2 (left)) while these oscillations disappeared when the standard mass matrix is replaced by the modified mass matrix, i.e. $M=M^{\text {mod }}$ (see Figure 2 (right)). However the approximated solutions obtained by both methods converge to the analytical solution when both the space $\Delta x$ and 
time $\Delta t$ steps tend to 0 in $\mathrm{L}^{p}(0, T ; \mathrm{H})$ norms with $p=2,+\infty$, see Figure 3 (a constant ratio $\frac{\Delta x}{\Delta t}=1$ has been used).
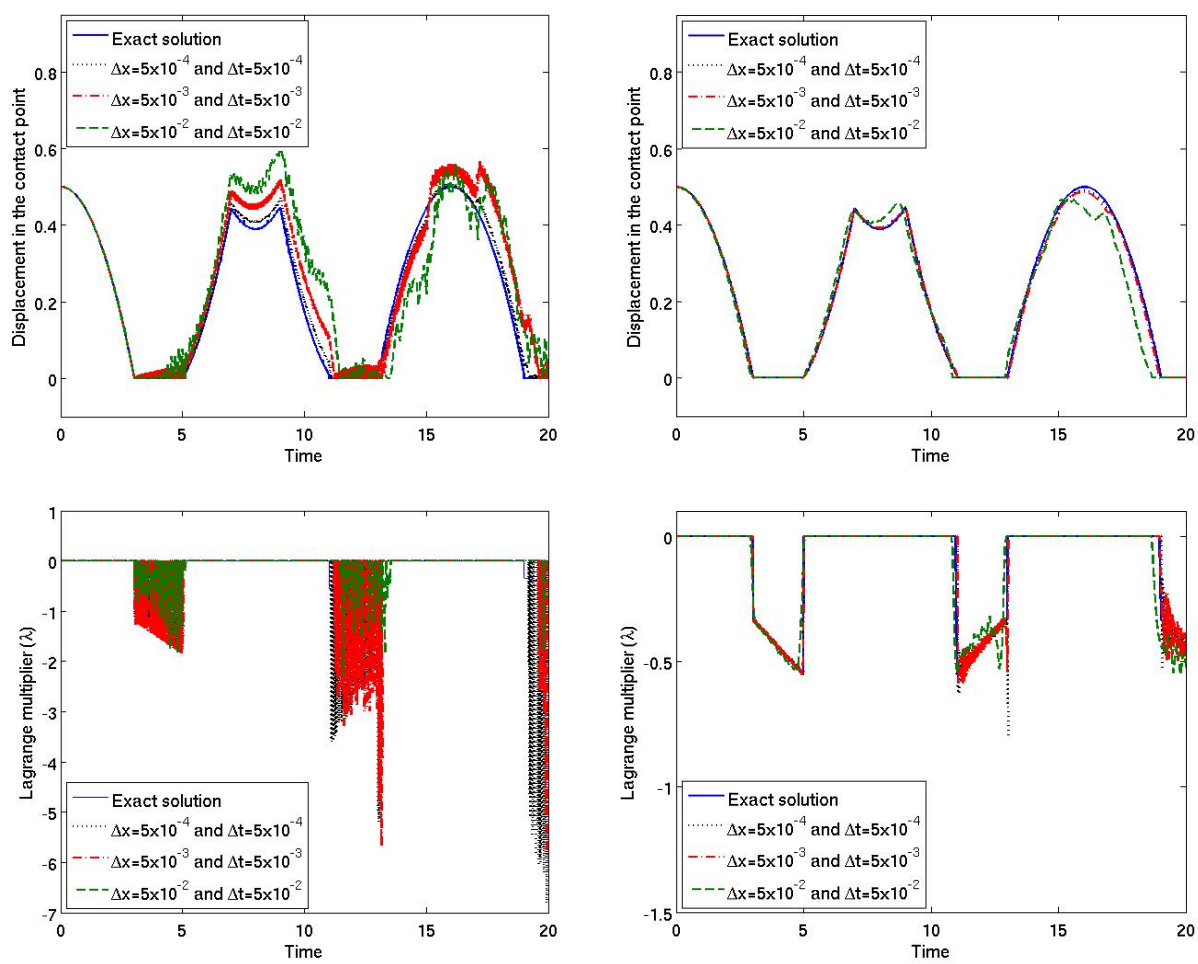

Figure 2: First analytical solution: comparison of the analytical solution $(u, \lambda)$ and approximated solutions $\left(u_{h}^{n}, \lambda_{n}\right)$ by using standard mass matrix (left) and modified mass matrix (right) in the contact with Crank-Nicolson method.

Surprisingly, the error curves in the norms $\left\|u_{h}-u\right\|_{\mathrm{L}^{p}(0, T ; \mathrm{V})}$, with $p=2,+\infty$ shown in the same figure are both diverging for the standard and modified mass matrices. The explanation of this divergence is illustrated in Figure 4 where the space derivative of the displacement is shown for both the analytical and the approximated solution. Indeed, Figure 4 (left) illustrates the fact that the series of the derivative corresponding to (37) do not converge. This is why it seems not to be possible to use this analytical solution to estimate the error in $\mathrm{L}^{p}(0, T ; \mathrm{V})$ norm. For that reason, another analytical solution is exhibited in the next section. 


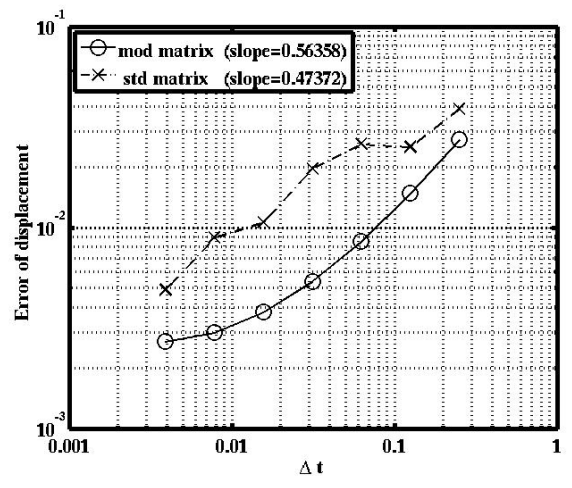

(a) $\left\|u_{h}-u\right\|_{\mathrm{L}^{\infty}(0, T ; \mathrm{H})}$

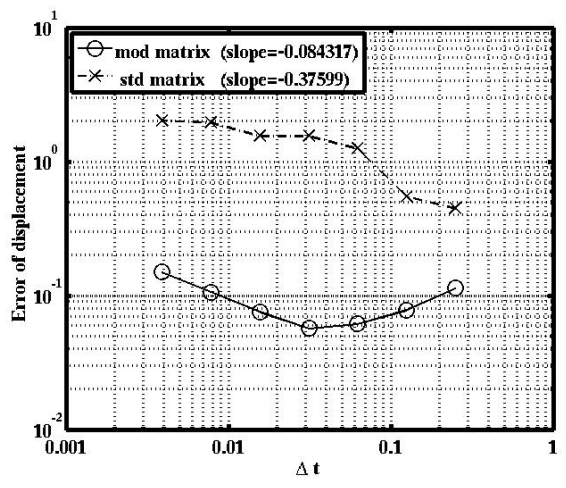

(c) $\left\|u_{h}-u\right\|_{\mathrm{L}^{\infty}(0, T ; \mathrm{V})}$

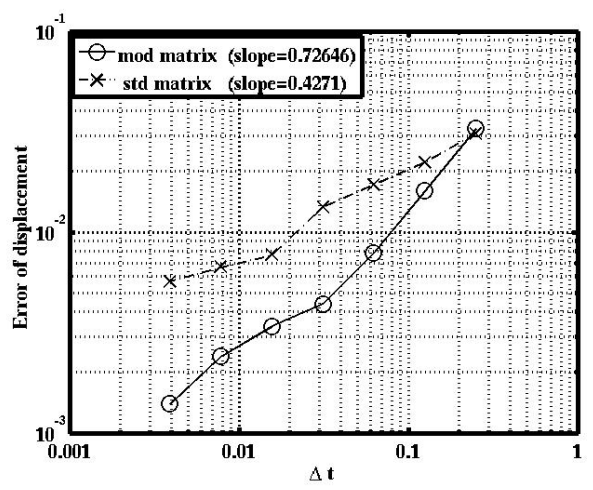

(b) $\left\|u_{h}-u\right\|_{\mathrm{L}^{2}(0, T ; \mathrm{H})}$

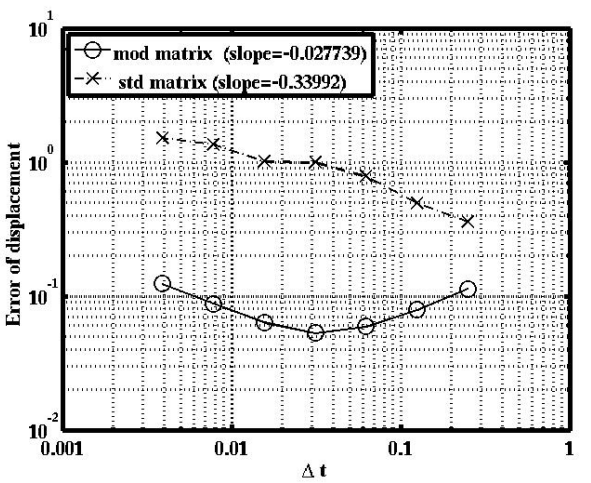

(d) $\left\|u_{h}-u\right\|_{\mathrm{L}^{2}(0, T ; \mathrm{V})}$

Figure 3: First analytical solution: comparison of the error curves obtained by using standard and modified mass matrices with Crank-Nicolson method. A constant ratio $\frac{\Delta x}{\Delta t}=1$ has been used. 

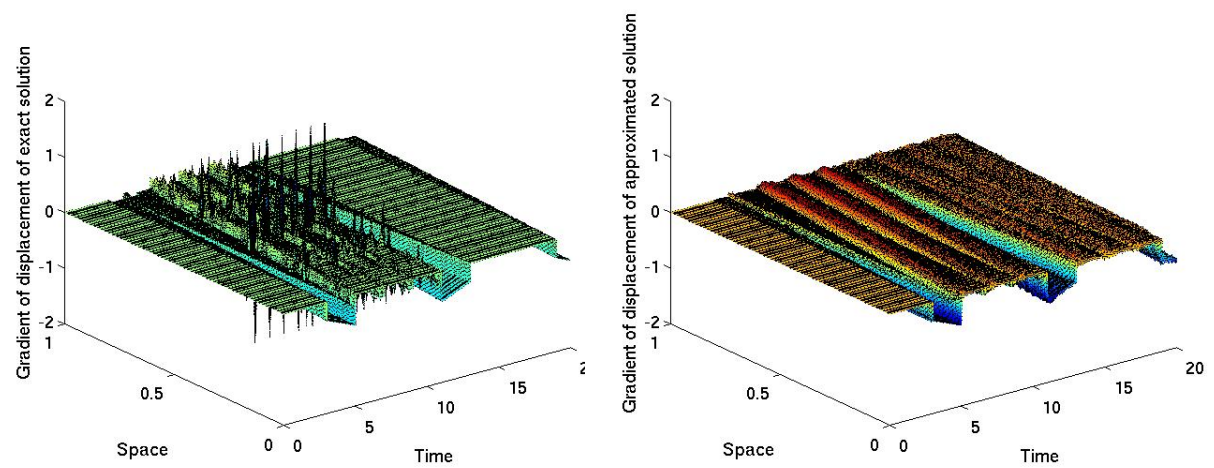

Figure 4: First analytical solution: representation of the derivative of displacement with respect to $x$ of analytical (left) and approximated (right) solutions. The analytical solution is computed with the $10^{3}$ first terms of the series.

\section{The wave equation with Signorini and Dirichlet boundary con- ditions}

\subsection{Analytical solution}

In this section, we propose a new analytical piecewise affine and periodic solution to the problem composed by the momentum equilibrium equation (1) together with Cauchy initial data (2) and boundary conditions (3) in the case where the density of external forces $f(x, t)$ vanishes. Suppose that the initial displacement and velocity are given by $u^{0}(x)=\frac{1}{2}-\frac{x}{2}$ and $v^{0}(x)=0$, respectively. The length of bar is $L=1$, namely, the bar is compressed at $t=0$. Let us describe the solution of this problem (see Figure 5). Before the impact, the bar, which is clamped at one end, elongates until it reaches the rigid obstacle at $t_{1}=1$. After the impact, the bar stays in contact for the time $t \in\left(t_{1}, t_{2}\right)$ and it takes off at time $t_{2}=2$.

We use characteristics method to determine the solution of the above problem. To this aim, we split each domain defined by $(0, L) \times\left(t_{i}, t_{i+1}\right), i=0,1,2$, corresponding to the phases before the impact, during the impact and after the impact, respectively, into four regions according to Figure 6 . We choose below $L=1$ and $t_{i}=i, i=0, \ldots, 3$.

Before the impact, according to characteristics lines $\xi \stackrel{\text { def }}{=} x+t$ and $\eta \stackrel{\text { def }}{=} x-t$, we divide the domain $(0,1) \times(0,1)$ into four regions as it is drawn on Figure 6 . Then we observe that $\xi \in[0,1]$ and $\eta \in[0,1]$ in the region $\mathrm{I}, \xi \in[0,1]$ and $\eta \in[-1,0]$ in the region II, $\xi \in[1,2]$ and $\eta \in[0,1]$ in the region III and 


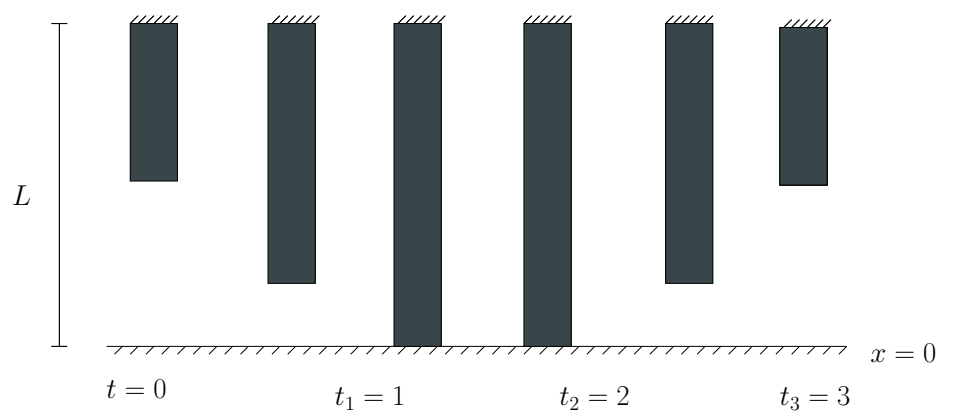

Figure 5: An elastic bar with one end fixed and the other end free to move

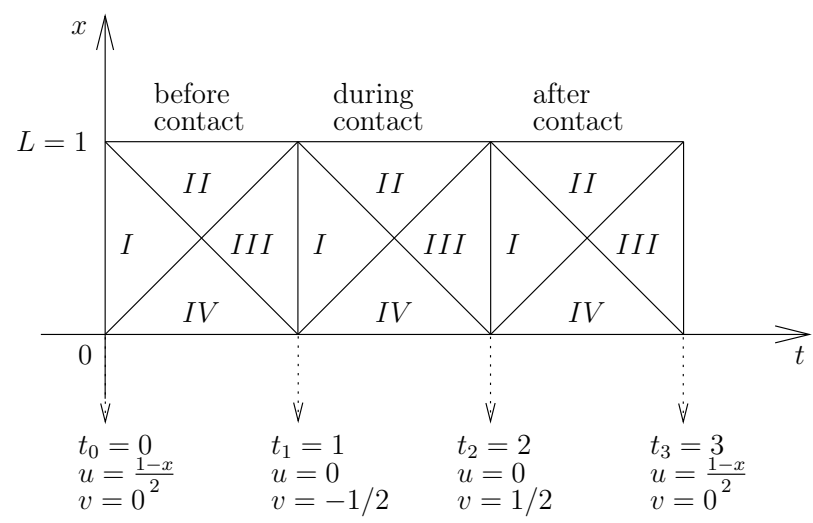

Figure 6: The regions allowing to determine the value of $\mathrm{u}$.

$\xi \in[1,2]$ and $\eta \in[-1,0]$ in the region IV. Therefore we may conclude that the solution is given by

$$
\begin{aligned}
& u(x, t)=\frac{1}{2}(1-x) \text { in the regions I, III, } \\
& u(x, t)=\frac{1}{2}(1-t) \text { in the regions II, IV. }
\end{aligned}
$$

Once again by using the characteristics lines, we divide the domain $(1,2) \times$ $(0,1)$ into four regions. Then the solution $(38 \mathrm{~b})$ calculated in the region IV allows us to evaluate the initial conditions at time $t_{1}=1$, namely we found $u(\cdot, 1)=0$ and $u_{t}(\cdot, 1)=-\frac{1}{2}$. Therefore we obtain

$$
\begin{array}{r}
u(x, t)=-\frac{1}{2}\left(t_{1}-t\right) \text { in the region } \mathrm{I}, \\
u(x, t)=-\frac{x}{2} \text { in the region II, } \\
u(x, t)=\frac{1}{2}(x-1) \text { in the region III, } \\
u(x, t)=\frac{1}{2}\left(t-t_{1}-1\right) \text { in the region IV. }
\end{array}
$$


After the impact, we divide the domain $(2,3) \times(0,1)$ into four regions. It follows from $(39 \mathrm{~d})$ that $u(\cdot, 2)=0$ and $u_{t}(\cdot, 2)=\frac{1}{2}$ which implies that

$$
\begin{aligned}
& u(x, t)=\frac{1}{2}\left(t-t_{2}\right) \text { in the regions I, II, } \\
& u(x, t)=\frac{1}{2}(1-x) \text { in the regions III, IV. }
\end{aligned}
$$

Furthermore we observe that $u(\cdot, 3)=u^{0}$ and $u_{t}(\cdot, 3)=v^{0}$. Then we conclude that the solution is periodic of period 3 . Note that the Lagrange multiplier $\lambda$ is equal to the contact force $u_{x}(0, \cdot)$.

\subsection{Numerical simulations}

We evaluate below $\left(U_{h}^{n}, \lambda^{n}\right)$ by using different time-stepping methods, namely the Newmark method (30), the backward Euler method (32) with $\theta=1$ and the Paoli-Schatzman method (34). More precisely, for each timestepping method, the approximated solutions $\left(U_{h}^{n}, \lambda^{n}\right)$ are obtained by solving (30) for different values of the couple $(\beta, \gamma)$, (34) for $\beta=\frac{1}{4}$ and different values for the parameter $e$ and (32) in the particular case where $\theta=1$, respectively. The computation are also performed for both the standard mass matrix $M$ and the modified one $M=M^{\text {mod }}$ and they are compared to the analytical solution $(u, \lambda)$ exhibited in Section 5.1 (see Figures 7, 11, 13, 15, 17, 19 and 21). Furthermore, the energy evolution associated to different methods mentioned above are presented in Figures 8, 12, 14, 16, 18, 20 and 22. Note that the numerical experiments were still done for different space and time steps with a constant ratio $\frac{\Delta x}{\Delta t}=10$. Some error curves for CrankNichoslon's method are presented for standard matrix as well as for modified mass matrix. Notice that the slope of error curves for nonlinear problems reflect both the regularity of solution and the ability of numerical schemes to reproduce some technical inequalities. In the Table 1 , we used the following notation: $\left\|u_{h}-u\right\|_{\mathrm{L}^{p}(\mathrm{H})}=\left\|u_{h}-u\right\|_{\mathrm{L}^{p}(0, T ; \mathrm{H})}$ with $p=2,+\infty$.

Finally, the convergence rates for different time-stepping methods, analyzed in details above, are summarized in Tables 1 and 2 for the reader convenience. These convergence rates were obtained for both standard (STD) and modified (MOD) mass matrices.

One can see in Table 1, that the rate of convergence of order one methods (Newmark I and backward Euler) in $\mathrm{L}^{p}(0, T ; \mathrm{H})$ norms of the displacement

(with $p=2,+\infty$ ) are limited to approximatively $\frac{1}{2}$ for both the standard and modified mass matrices. Concerning the other methods, which are order two methods at least for the linear part of the problem, the rate of 


\begin{tabular}{|c|c|c|c|c|c|}
\hline Scheme & Method & $\left\|u_{h}-u\right\|_{\mathrm{L}^{\infty}(\mathrm{H})}$ & $\left\|u_{h}-u\right\|_{\mathrm{L}^{2}(\mathrm{H})}$ & $\left\|u_{h}-u\right\|_{\mathrm{L}^{\infty}(\mathrm{V})}$ & $\left\|u_{h}-u\right\|_{\mathrm{L}^{2}(\mathrm{~V})}$ \\
\hline \multirow{2}{*}{ Crank-Nicolson } & STD & 0.48045 & 0.47113 & -0.30273 & -0.31299 \\
& MOD & 0.88075 & 0.97113 & 0.38624 & 0.36192 \\
\hline Newmark I & STD & 0.51444 & 0.51768 & 0.26104 & 0.24000 \\
$(\beta, \gamma)=\left(\frac{1}{2}, 1\right)$ & MOD & 0.49236 & 0.48034 & 0.28219 & 0.25943 \\
\hline Newmark II & STD & 0.57503 & 0.61856 & 0.34411 & 0.30100 \\
$(\beta, \gamma)=\left(\frac{1}{2}, \frac{1}{2}\right)$ & MOD & 0.95055 & 1.00090 & 0.39734 & 0.33789 \\
\hline Backward Euler & STD & 0.51804 & 0.51771 & 0.26784 & 0.24847 \\
\hline Paoli-Schatzman I & MOD & 0.50425 & 0.49024 & 0.27753 & 0.25465 \\
\hline$e=0$ & STD & 0.66064 & 0.66015 & 0.31204 & 0.28406 \\
\hline Paoli-Schatzman II & STD & 0.66064 & 0.66014 & 0.33914 & 0.41096 \\
\hline$e=\frac{1}{2}$ & MOD & 0.97659 & 1.00170 & 0.41159 & 0.42823 \\
\hline Paoli-Schatzman III & STD & 0.58876 & 0.61110 & 0.39286 & 0.35512 \\
$e=1$ & MOD & 0.97658 & 1.00200 & 0.46380 & 0.46993 \\
\hline
\end{tabular}

Table 1: Convergence rates for the displacement

\begin{tabular}{|c|c|c|c|c|c|}
\hline Scheme & Method & $\left\|\lambda_{h}-\lambda\right\|_{\mathrm{L}^{2}(0, T)}$ & Oscillations & $\left\|\mathcal{E}_{h}-\mathcal{E}\right\|_{\mathrm{L}^{\infty}(0, T)}$ & $\left\|\mathcal{E}_{h}-\mathcal{E}\right\|_{\mathrm{L}^{2}(0, T)}$ \\
\hline \multirow{2}{*}{ Crank-Nicolson } & STD & -0.13546 & Big & -0.61885 & -0.60737 \\
& MOD & 0.48812 & Small & 0.99486 & 0.99313 \\
\hline Newmark I & STD & 0.01366 & Yes & 0.50094 & 0.49943 \\
$(\beta, \gamma)=\left(\frac{1}{2}, 1\right)$ & MOD & 0.31778 & No & 0.51230 & 0.49687 \\
\hline Newmark II & STD & -0.01996 & Big & 1.05720 & 1.08500 \\
$(\beta, \gamma)=\left(\frac{1}{2}, \frac{1}{2}\right)$ & MOD & 0.49128 & Small & 0.99887 & 1.00790 \\
\hline Backward Euler & STD & 0.25559 & No & 0.50055 & 0.49963 \\
\hline Paoli-Schatzman I & MOD & 0.30221 & No & 0.50918 & 0.49803 \\
\hline$e=0$ & MOD & 0.28976 & Yes & 1.01620 & 1.02650 \\
\hline Paoli-Schatzman II & STD & 0.30798 & Yes & 0.99331 & 1.00380 \\
\hline$e=\frac{1}{2}$ & MOD & 0.35044 & Yes & 1.33470 & 1.00160 \\
\hline Paoli-Schatzman III & STD & 0.04596 & Yes & 1.02640 & 0.99142 \\
$e=1$ & MOD & 0.34364 & Yes & 1.01270 & 1.03150 \\
\hline
\end{tabular}

Table 2: Convergence rates for the Lagrange multiplier and energy

convergence is still approximatively $\frac{1}{2}$ for the standard mass matrix but is improved to approximatively 1 for the modified mass matrix. Note that the Paoli-Schatzman scheme is not a fully order two method, except for $e=1$, 
due to the discretization of the contact condition.

The advantage is less pronounced in $\mathrm{L}^{p}(0, T$; V) norms of the displacement, but it is even more important in $\mathrm{L}^{2}(0, T)$ norm of the contact stress (see Table 2) since convergence does not occur for all the methods with the standard mass matrix.

An important remark is that, despite the low regularity of the exact solution, order two methods perform better than order one methods and should be preferred to approximate elastodynamic problem with impact.

Concerning the efficiency of the mass redistribution method, we can conclude that, even though it represents an additional approximation compared to the standard approximation, the convergence rates on the displacement and on the contact force are improved and the method reduces the potential spurious oscillations. The mass redistribution method appears then to be a robust method to approximate elastodynamic problem with impact.

\subsubsection{The Crank-Nicolson method}

We focus here on Newmark's method (30) in the particular case where $(\beta, \gamma)=\left(\frac{1}{4}, \frac{1}{2}\right)$.

The analytical solution $(u, \lambda)$ exhibited in Section 5.1 and the approximated solutions $\left(U_{h}^{n}, \lambda^{n}\right)$ obtained for different time and space steps are represented on Figure 7. We observe some spurious oscillations on Figure 7 (left) for the solution $\left(U_{h}^{n}, \lambda^{n}\right)$ to Problem (30) after the contact takes place while these oscillations disappear when the standard mass matrix is replaced by the modified one, see Figure 7 (right).

It can easily be seen on Figure 8 that the scheme with the standard mass matrix is unstable with a rapidly growing energy while the one with the modified mass matrix is almost conservative as the space $\Delta x$ and time $\Delta t$ steps tend to 0 . The error curves for $\left(U_{h}^{n}, \lambda^{n}\right)$ highlighted that the norms $\left\|u_{h}-u\right\|_{\mathrm{L}^{p}(0, T ; \mathrm{H})}$, with $p=2,+\infty$, and $\left\|\lambda^{n}-\lambda\right\|_{\mathrm{H}}$ converge to 0 when $M=M^{\text {mod }}$ as $n$ tends to $+\infty$, which is unfortunately not the case for the standard mass matrix (see Figure 10). Finally, Figure 9 shows that the norm $\left\|\mathcal{E}_{h}-\mathcal{E}\right\|_{\mathrm{L}^{p}(0, T)}, p=2,+\infty$, converges when $M=M^{\text {mod }}$ while this norm diverges when the standard mass matrix is considered. 

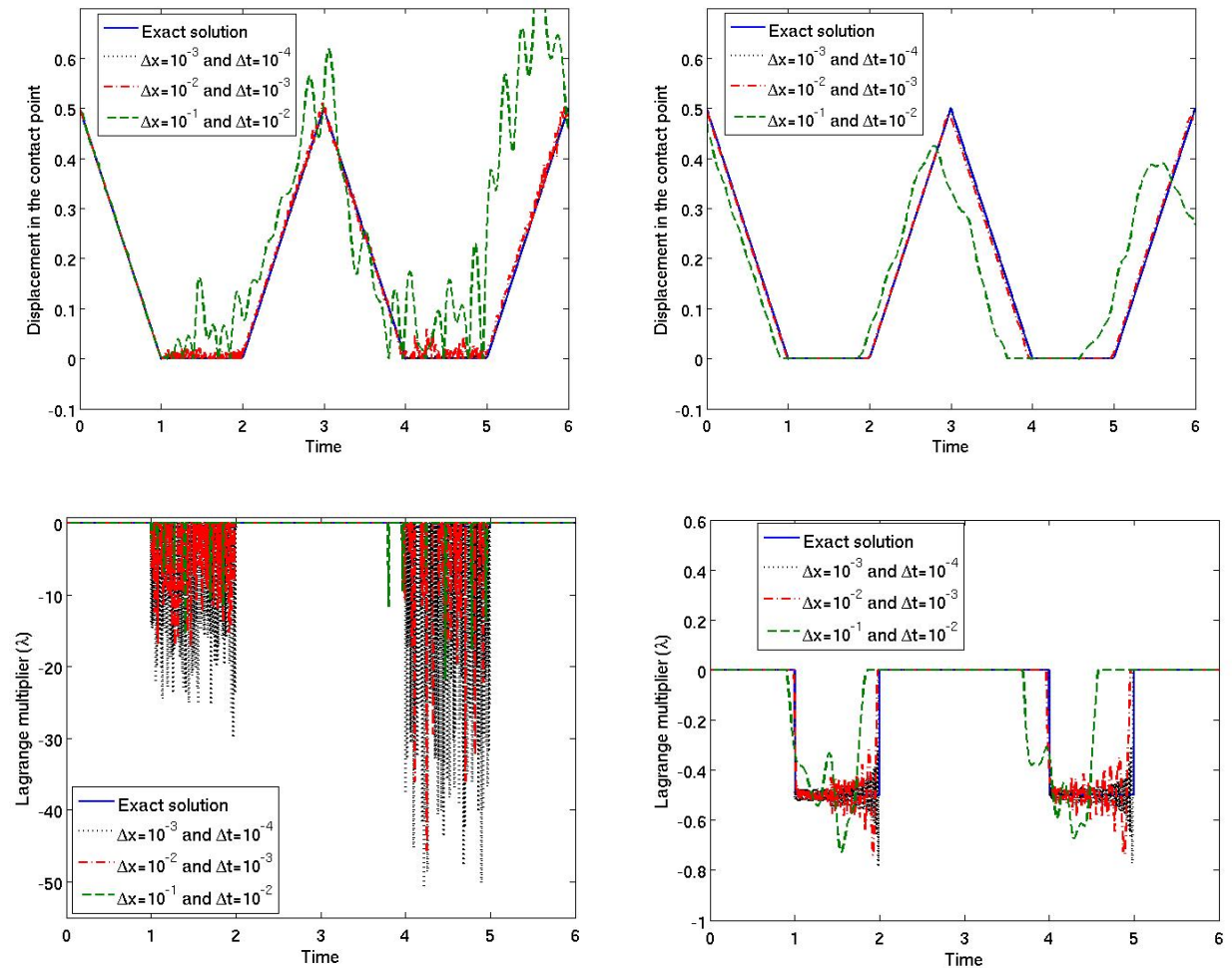

Figure 7: Comparison of the analytical solution $(u, \lambda)$ and the approximated solutions $\left(U_{h}^{n}, \lambda^{n}\right)$ by using the standard (left) and modified (right) mass matrices in the contact node with Crank-Nicolson method.
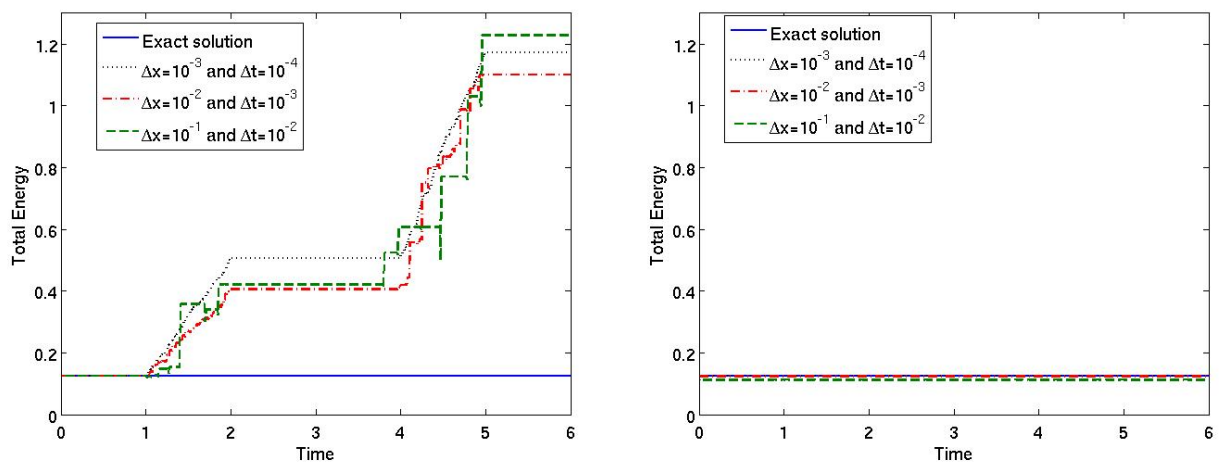

Figure 8: Comparison of the energy associated to the analytical solution and the energy associated to the approximated ones for the standard (left) and modified (right) mass matrices with Crank-Nicolson method. 


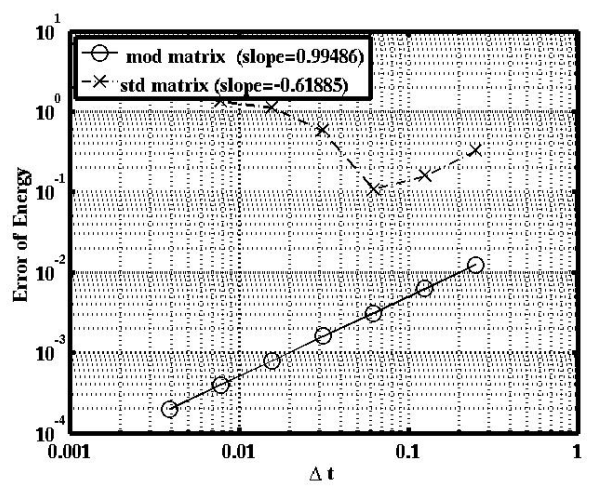

(a) $\left\|\mathcal{E}_{h}-\mathcal{E}\right\|_{L^{\infty}(0, T)}$

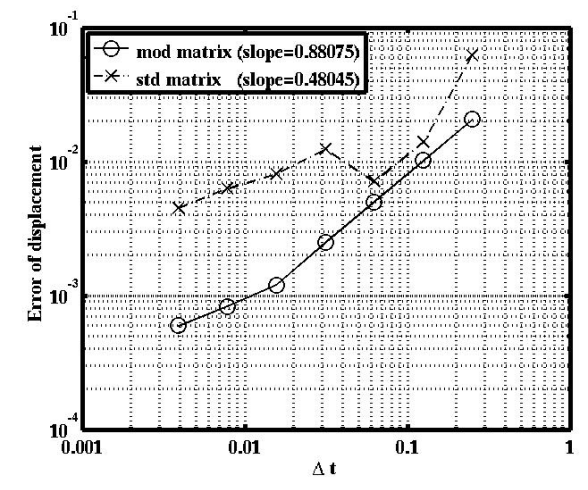

(c) $\left\|u_{h}-u\right\|_{\mathrm{L}^{\infty}(0, T ; \mathrm{H})}$

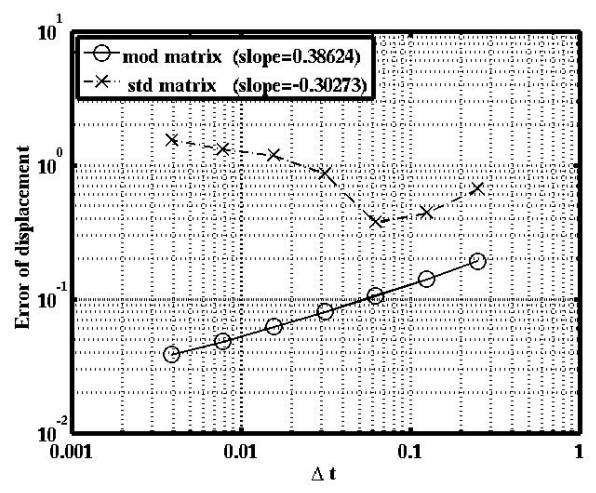

(e) $\left\|u_{h}-u\right\|_{\mathrm{L}^{\infty}(0, T ; \mathrm{V})}$

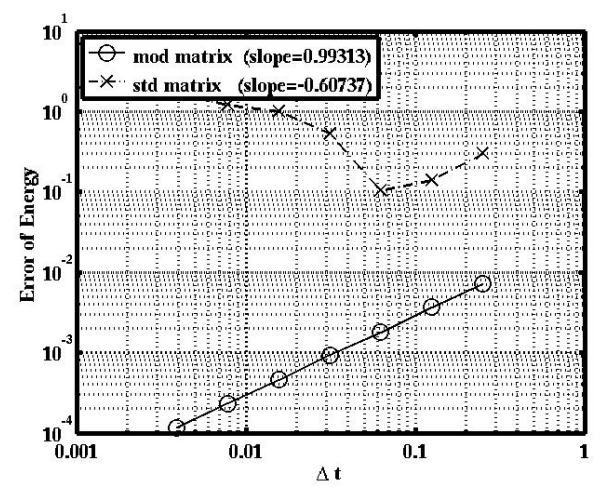

(b) $\left\|\mathcal{E}_{h}-\mathcal{E}\right\|_{\mathrm{L}^{2}(0, T)}$

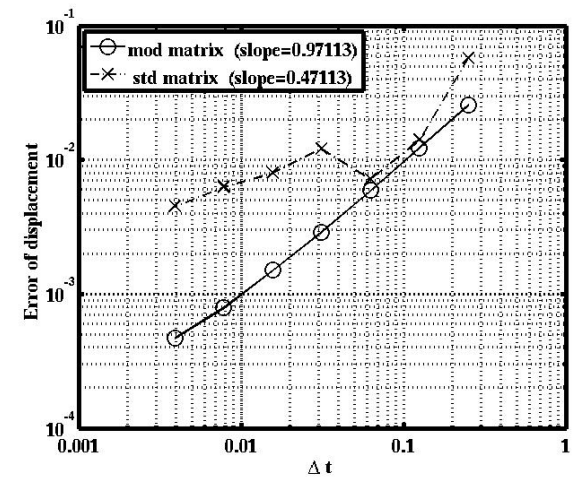

(d) $\left\|u_{h}-u\right\|_{\mathrm{L}^{2}(0, T ; \mathrm{H})}$

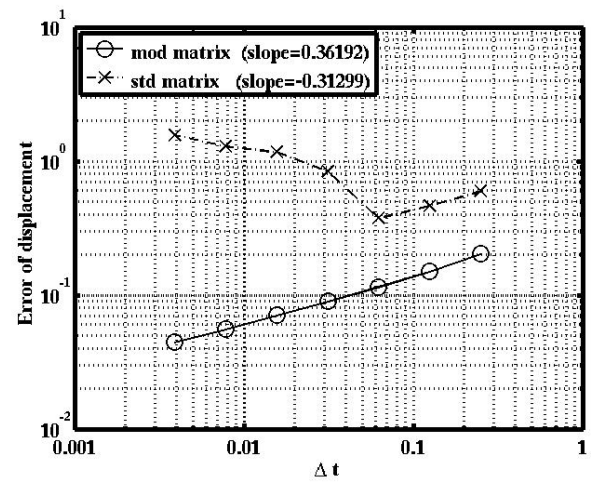

(f) $\left\|u_{h}-u\right\|_{\mathrm{L}^{2}(0, T ; \mathrm{V})}$

Figure 9: Comparison of the error curves of the energy obtained by using the standard and modified mass matrices with Crank-Nicolson method. 


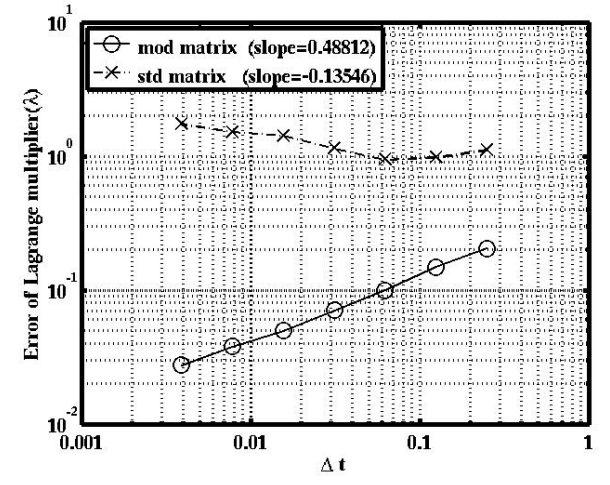

(a) $\left\|\lambda^{n}-\lambda\right\|_{\mathrm{L}^{2}(0, T)}$

Figure 10: Comparison of the error curves obtained by using the standard and modified mass matrices with Crank-Nicolson method.

\subsubsection{The Newmark method I}

We deal now with Newmark's method (30) in the particular case where $(\beta, \gamma)=\left(\frac{1}{2}, 1\right)$. We observe some oscillations on Figure 11 (left) for the Lagrange multiplier $\lambda^{n}$ associated to Problem (30) after the contact takes place while these oscillations are much less preeminent when the standard mass matrix is replaced by the modified one, see Figure 11 (right). Furthermore, the present scheme is dissipative and stable.
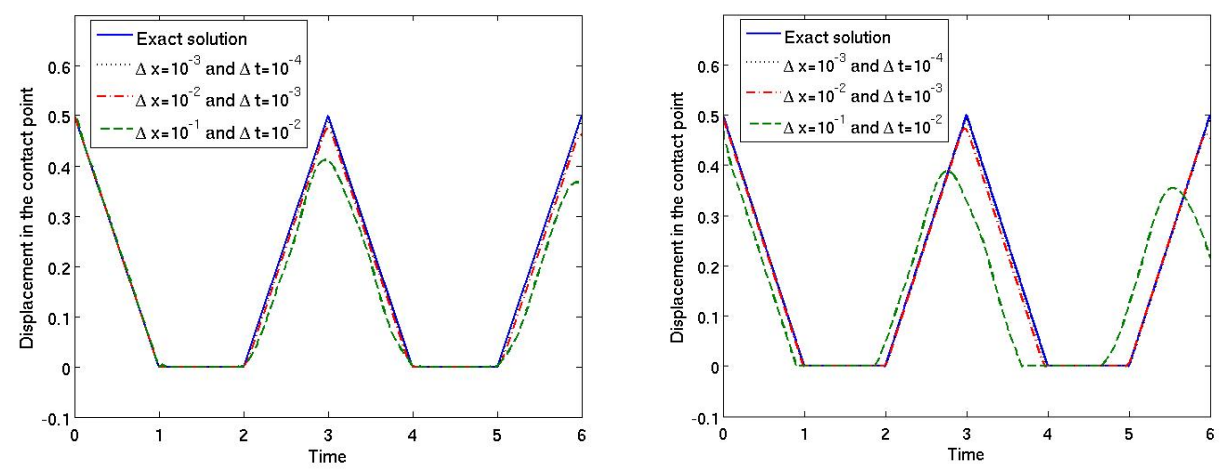

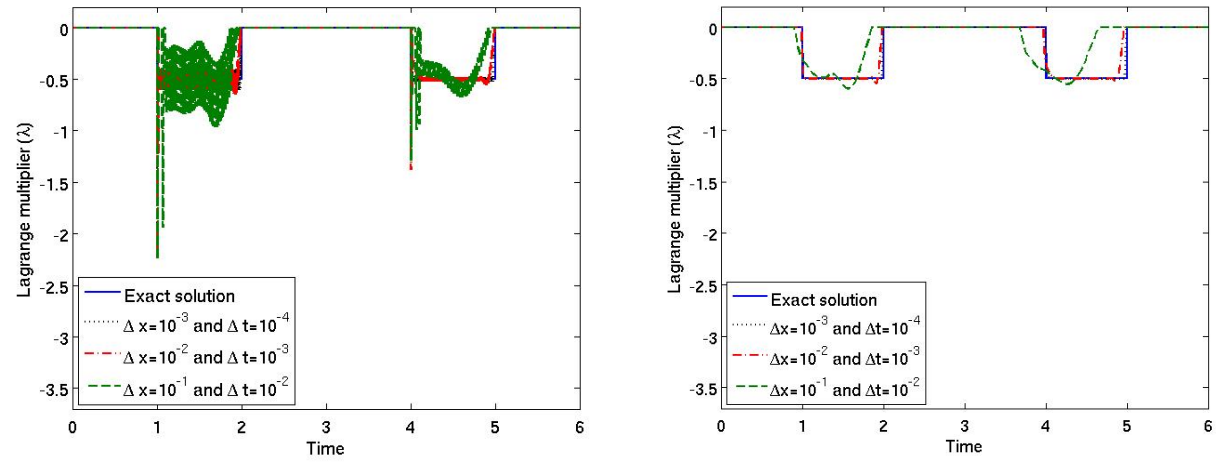

Figure 11: Comparison of the analytical solution $(u, \lambda)$ and the approximated solutions $\left(U_{h}^{n}, \lambda^{n}\right)$ by using the standard (left) and modified (right) mass matrices in the contact with Newmark method I.
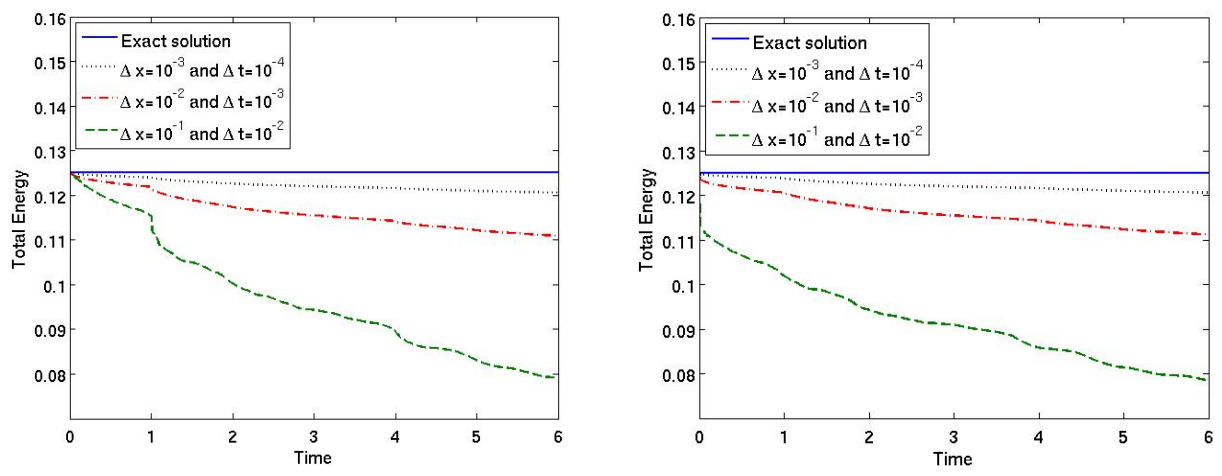

Figure 12: Comparison of the energy associated to the analytical solution and the energy associated to the approximated ones for standard (left) and modified (right) mass matrices with Newmark method I.

\subsubsection{The Newmark method II}

We consider Newmark's method (30) in the case where $(\beta, \gamma)=\left(\frac{1}{2}, \frac{1}{2}\right)$. Some oscillations on Figure 13 (left) for the Lagrange multiplier $\lambda^{n}$ associated to Problem (30) can be observed after the contact takes place while these oscillations are much less important when the modified mass matrix is considered in (30), see Figure 13 (right). Note that here the scales for the Lagrange multiplier associated to standard and modified mass matrices are different, see Figure 13. 

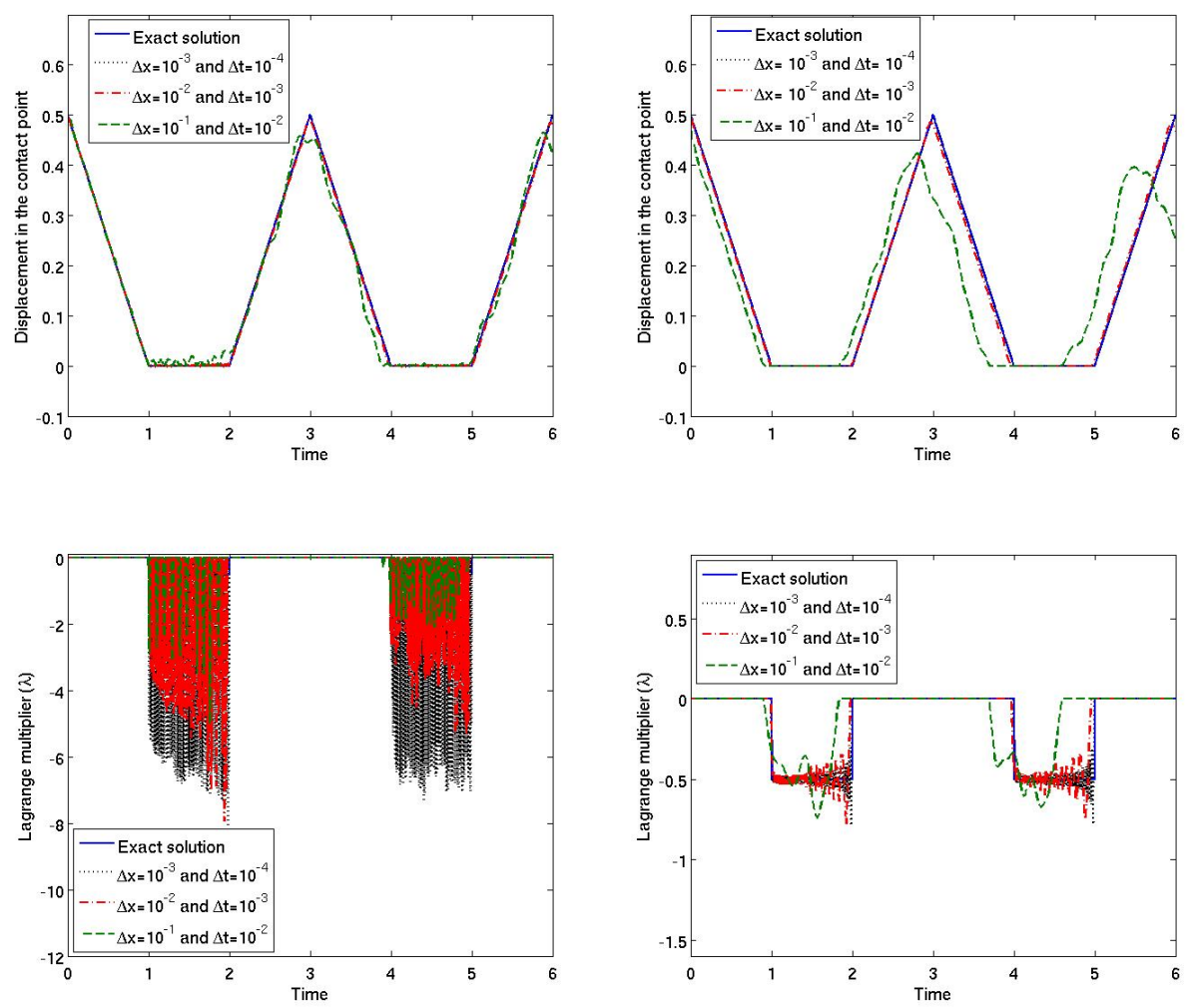

Figure 13: Comparison of the analytical solution $(u, \lambda)$ and the approximated solutions $\left(U_{h}^{n}, \lambda^{n}\right)$ by using the standard (left) and modified mass matrices (right) in the contact with Newmark method II.
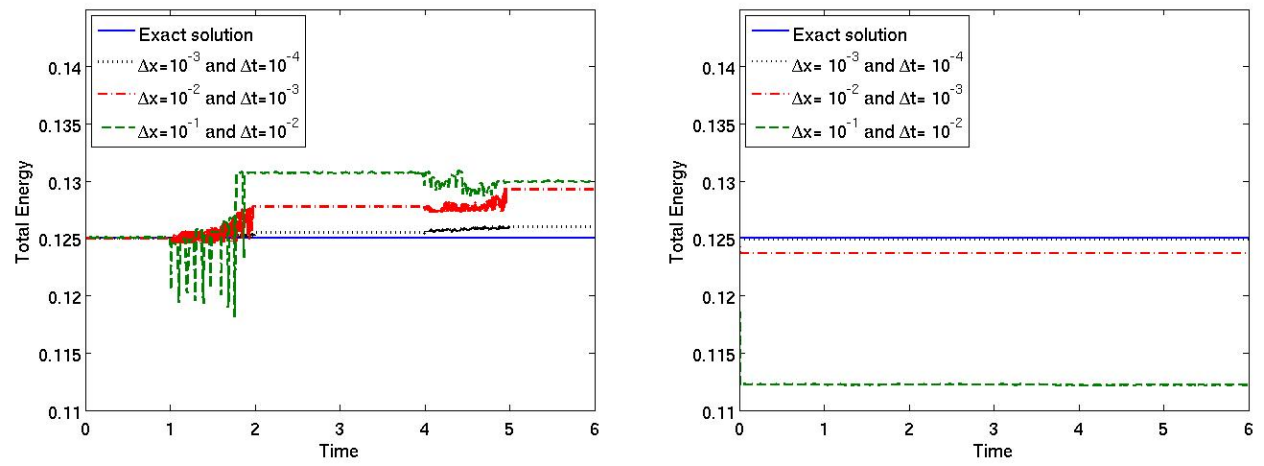

Figure 14: Comparison of the energy associated to the analytical solution and the energy associated to the approximated ones for the standard (left) and modified (right) mass matrices with Newmark method II. 


\subsubsection{The backward Euler method}

We deal here with backward Euler's method (i.e. (32) with $\theta=1$ ). Since the present scheme is dissipative and stable $\left(\Delta \mathcal{E}_{h}^{n}<0\right.$, see (33)), the approximated solutions $\left(U_{h}^{n}, \lambda^{n}\right)$ obtained by using the standard mass as well as modified mass matrices do not oscillate as it can be observed in the previous numerical simulations.
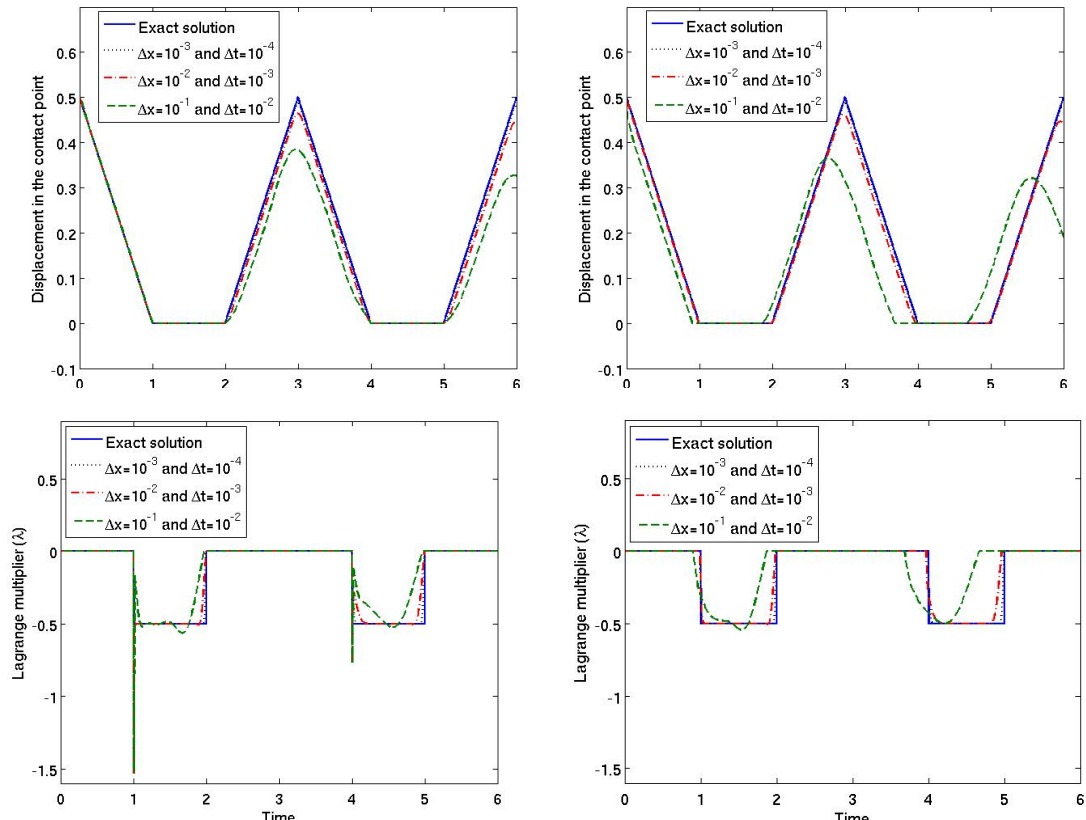

Figure 15: Comparison of the analytical solution $(u, \lambda)$ and the approximated solutions $\left(U_{h}^{n}, \lambda^{n}\right)$ by using the standard (left) and modified (right) mass matrices in the contact with backward Euler method.
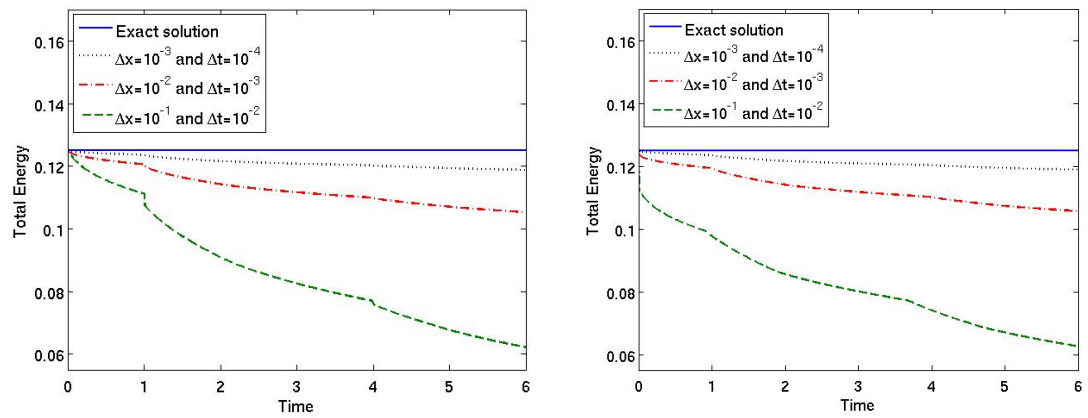

Figure 16: Comparison of the energy associated to the analytical solution and the energy associated to the approximated ones for the standard (left) and modified (right) mass matrices with backward Euler method. 


\subsubsection{The Paoli-Schatzman method I}

We are interested here in Paoli-Schatzman's method with $(\beta, e)=\left(\frac{1}{4}, 0\right)$. Note that the stability result straightforwardly follows from [8].
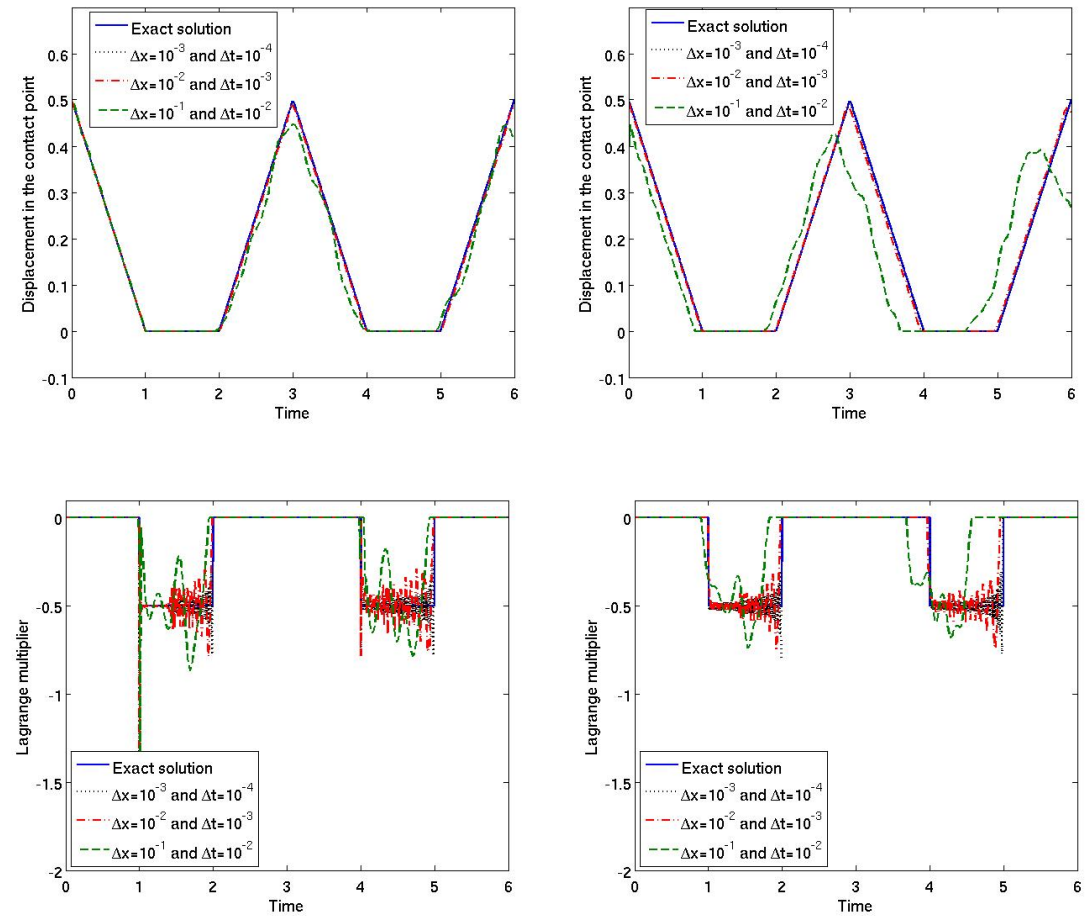

Figure 17: Comparison of the analytical solution $(u, \lambda)$ and the approximated solutions $\left(U_{h}^{n}, \lambda^{n}\right)$ by using the standard (left) and modified (right) mass matrices in the contact with Paoli-Schatzman method I.
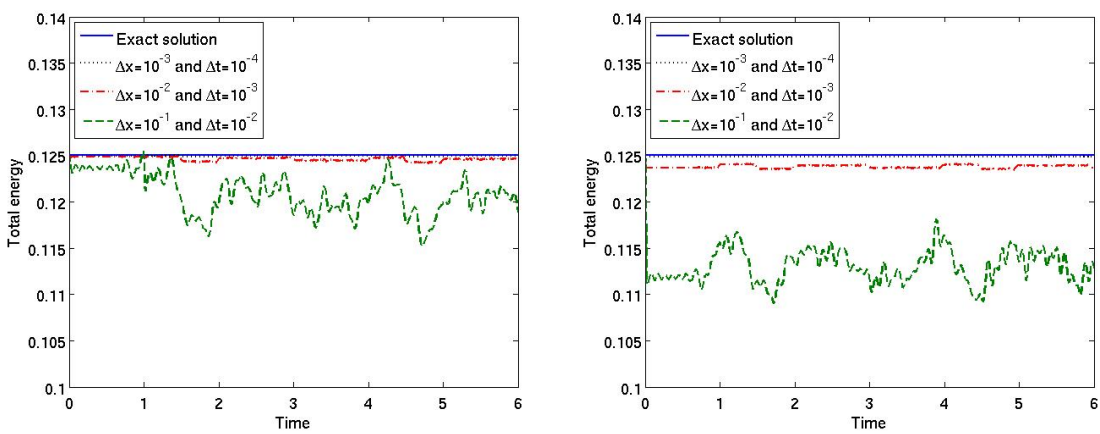

Figure 18: Comparison of the energy associated to the analytical solution and the energy associated to the approximated ones for the standard (left) and modified (right) mass matrices with Paoli-Schatzman method I. 


\subsubsection{The Paoli-Schatzman method II}

We focus here on Paoli-Schatzman's method with $(\beta, e)=\left(\frac{1}{4}, \frac{1}{2}\right)$. Observe that the stability result is still an open problem for $e \neq 0$. Some spurious oscillations can be observed for the approximated Lagrange multiplier as well as for the approximated energy.
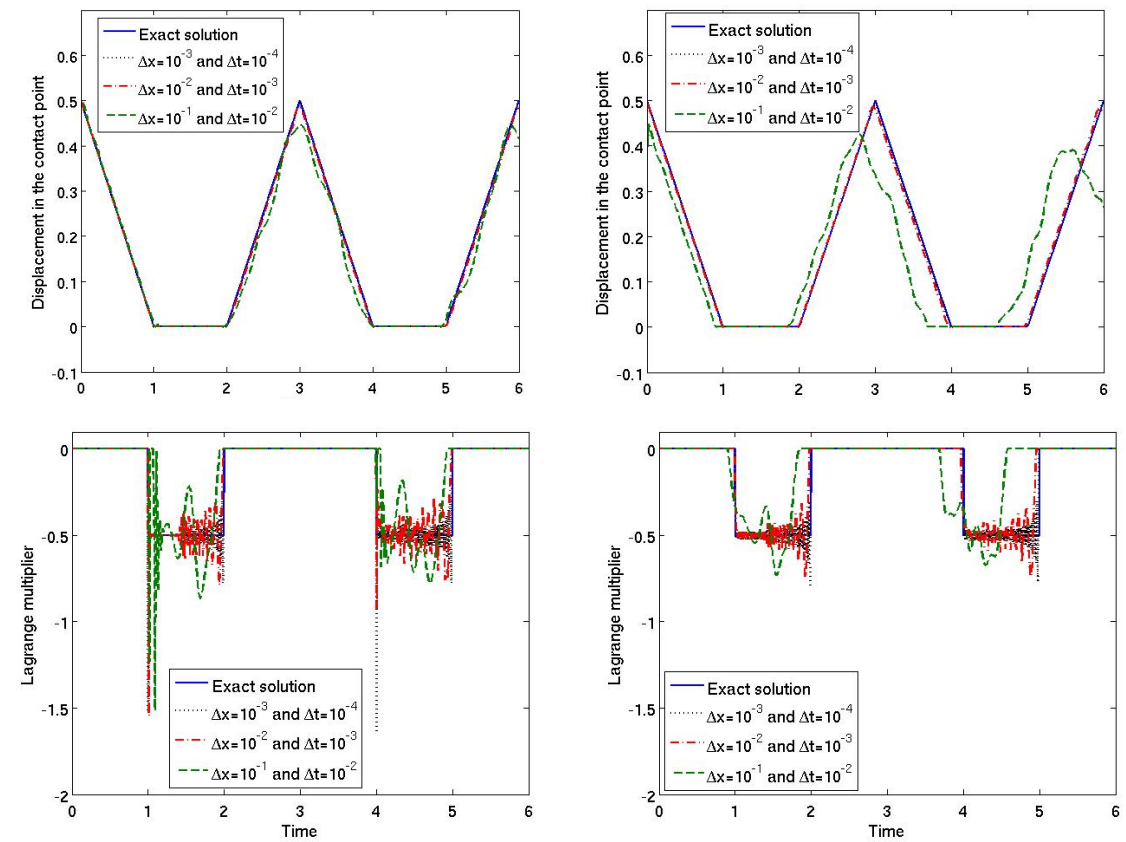

Figure 19: Comparison of the analytical solution $(u, \lambda)$ and the approximated solutions $\left(U_{h}^{n}, \lambda^{n}\right)$ by using the standard (left) and modified (right) mass matrices in the contact with Paoli-Schatzman method II.
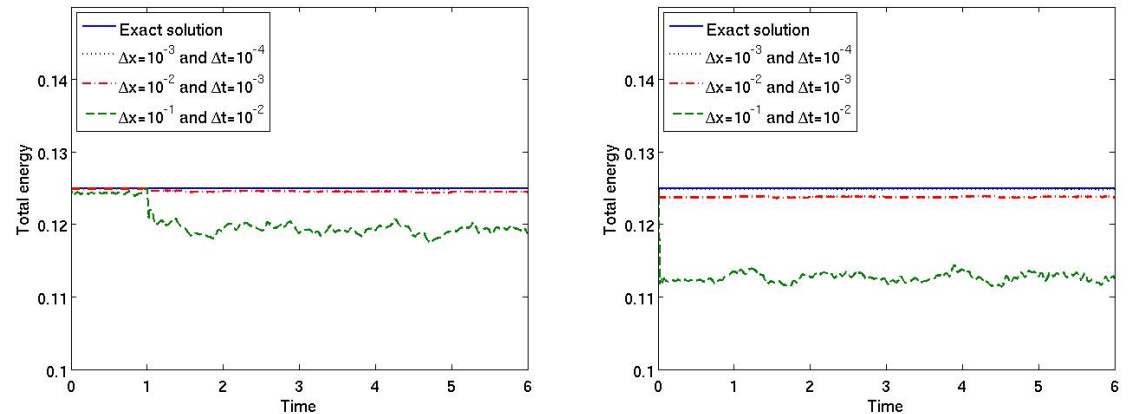

Figure 20: Comparison of the energy associated to the analytical solution and the energy associated to the approximated ones for the standard (left) and modified (right) mass matrices with Paoli-Schatzman method II. 


\subsubsection{The Paoli-Schatzman method III}

We consider now Paoli-Schatzman's method with $(\beta, e)=\left(\frac{1}{4}, 1\right)$. Note that this is a second order scheme.
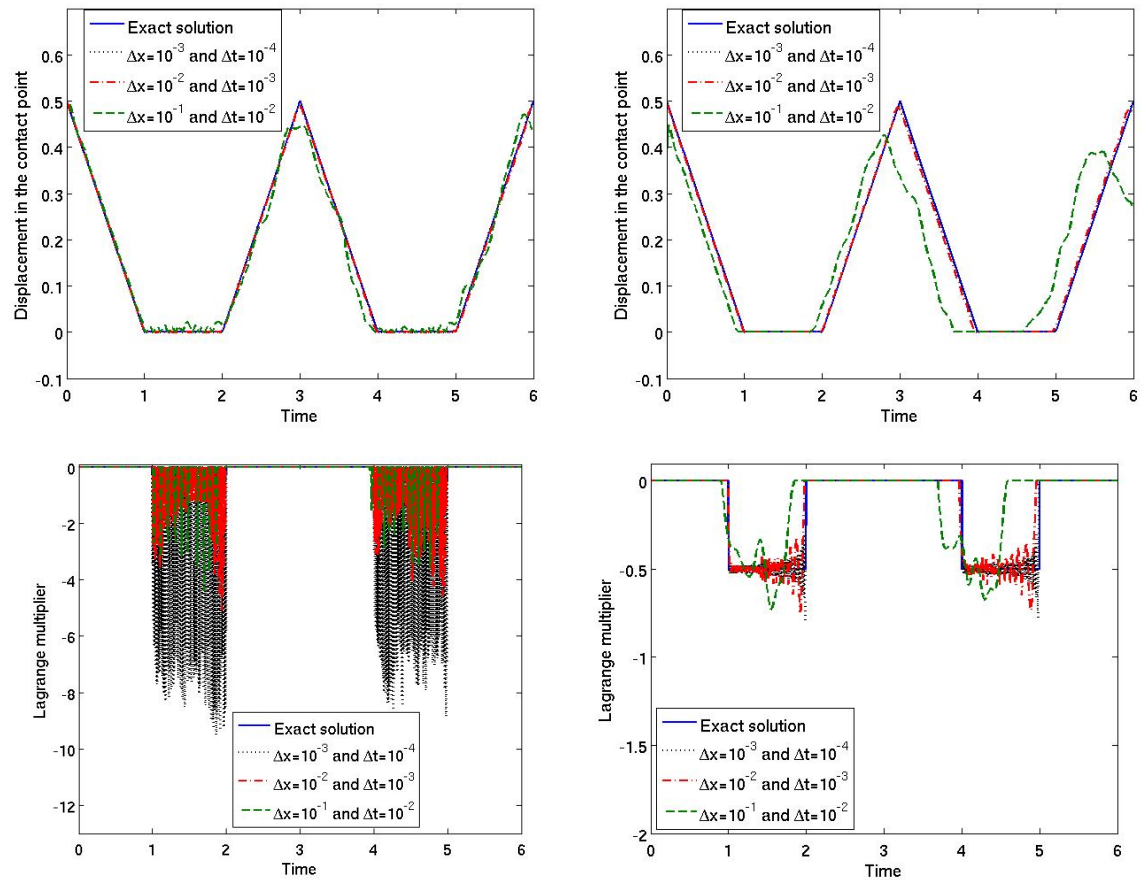

Figure 21: Comparison of the analytical solution $(u, \lambda)$ and the approximated solutions $\left(U_{h}^{n}, \lambda^{n}\right)$ by using standard (left) and modified (right) mass matrices in the contact with Paoli-Schatzman method III.
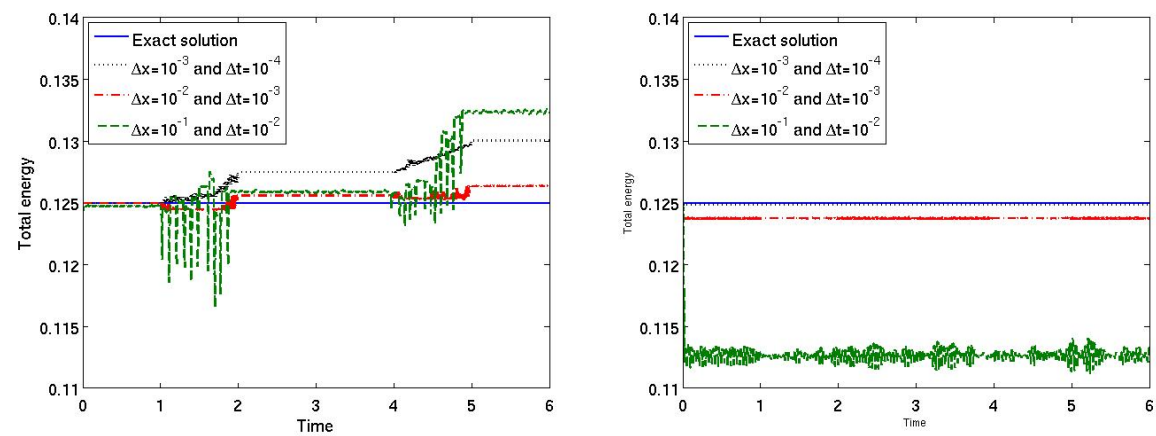

Figure 22: Comparison of the energy associated to the analytical solution and the energy associated to the approximated ones for the standard (left) and modified (right) mass matrices with Paoli-Schatzman method III. 
Acknowledgments. The support of the AVCR-CNRS "Mathematical and numerical analysis of contact problems for materials with memory" grant is gratefully acknowledged.

\section{Appendix}

The aim of the present section consists to establish the energy evolutions associated to schemes (30), (32) and (34). Let us observe that the energy evolution, when the external force $f=0$, is given by

$$
\Delta \mathcal{E}_{h}^{n}=\frac{1}{2}\left(U_{h, t}^{n+1}-U_{h, t}^{n}\right)^{\top} M\left(U_{h, t}^{n+1}+U_{h, t}^{n}\right)+\frac{1}{2}\left(U_{h}^{n+1}-U_{h}^{n}\right)^{\top} S\left(U_{h}^{n+1}+U_{h}^{n}\right) .
$$

We prove now that the energy evolution associated to the Crank-Nicolson method is given by(31). To this aim, we observe that (30a) and (30b) lead to

$$
\begin{aligned}
& M\left(U_{h}^{n+1}-U_{h}^{n}-\Delta t U_{h, t}^{n}\right)= \\
& -\Delta t^{2}\left(\left(\frac{1}{2}-\beta\right) S U_{h}^{n}+\beta S U_{h}^{n+1}\right)-\Delta t^{2}\left(\left(\frac{1}{2}-\beta\right) \lambda^{n} e_{0}+\beta \lambda^{n+1} e_{0}\right),
\end{aligned}
$$

and

$$
M\left(U_{h, t}^{n+1}-U_{h, t}^{n}\right)=-\Delta t\left((1-\gamma) S U_{h}^{n}+\gamma S U_{h}^{n+1}\right)-\Delta t\left((1-\gamma) \lambda^{n} e_{0}+\gamma \lambda^{n+1} e_{0}\right)
$$

Then we multiply (42) by $\frac{\Delta t}{2}$ and we subtract this expression to (41), we get

$$
\begin{aligned}
& M\left(U_{h, t}^{n+1}+U_{h, t}^{n}\right)=\frac{2}{\Delta t} M\left(U_{h}^{n+1}-U_{h}^{n}\right) \\
& +2 \Delta t\left(\left(\beta-\frac{\gamma}{2}\right) S\left(U_{h}^{n+1}-U_{h}^{n}\right)\right)+2 \Delta t\left(\left(\beta-\frac{\gamma}{2}\right)\left(\lambda^{n+1} e_{0}-\lambda^{n} e_{0}\right)\right) .
\end{aligned}
$$

By using (43) in (40), it follows that

$$
\begin{aligned}
& \Delta \mathcal{E}_{h}^{n}= \\
& \frac{1}{\Delta t}\left(U_{h, t}^{n+1}-U_{h, t}^{n}\right)^{\top} M\left(U_{h}^{n+1}-U_{h}^{n}\right)+\Delta t\left(\beta-\frac{\gamma}{2}\right)\left(U_{h, t}^{n+1}-U_{h, t}^{n}\right)^{\top} S\left(U_{h}^{n+1}-U_{h}^{n}\right) \\
& \left.+\Delta t\left(\beta-\frac{\gamma}{2}\right)\left(U_{h, t}^{n+1}-U_{h, t}^{n}\right)^{\top} \lambda^{n+1} e_{0}-\lambda^{n} e_{0}\right)+\frac{1}{2}\left(U_{h}^{n+1}-U_{h}^{n}\right)^{\top} S\left(U_{h}^{n+1}+U_{h}^{n}\right) .
\end{aligned}
$$


Since $M$ is a symmetric matrix, it follows from (42) that

$$
\begin{aligned}
\Delta \mathcal{E} & =\left(\frac{1}{2}-\gamma\right)\left(U_{h}^{n+1}-U_{h}^{n}\right)^{\top} S\left(U_{h}^{n+1}-U_{h}^{n}\right) \\
& -\left(U_{h}^{n+1}-U_{h}^{n}\right)^{\top}\left((1-\gamma) \lambda^{n} e_{0}+\gamma \lambda^{n+1} e_{0}\right) \\
& +\Delta t\left(\beta-\frac{\gamma}{2}\right)\left(U_{h, t}^{n+1}-U_{h, t}^{n}\right)^{\top} S\left(U_{h}^{n+1}-U_{h}^{n}\right) \\
& +\Delta t\left(\beta-\frac{\gamma}{2}\right)\left(U_{h, t}^{n+1}-U_{h, t}^{n}\right)^{\top}\left(\lambda^{n+1} e_{0}-\lambda^{n} e_{0}\right) .
\end{aligned}
$$

By setting the parameters $(\beta, \gamma)=\left(\frac{1}{4}, \frac{1}{2}\right)(31)$ holds.

Let us establish the energy evolution associated to the $\theta$-method. We infer from (32a) and (32c) that

$$
M\left(U_{h, t}^{n+1}-U_{h, t}^{n}\right)=(1-\theta) \Delta t\left(-S U_{h}^{n}-\lambda^{n} e_{0}\right)+\theta \Delta t\left(-S U_{h}^{n+1}-\lambda^{n+1} e_{0}\right) .
$$

Then using (44) in (40), we find

$$
\begin{aligned}
& \Delta \mathcal{E}_{h}^{n}=-\frac{\Delta t}{2}\left(U_{h, t}^{n+1}+U_{h, t}^{n}\right)^{\top}\left((1-\theta) S U_{h}^{n}+\theta S U_{h}^{n+1}+\left((1-\theta) \lambda^{n}+\theta \lambda^{n+1}\right) e_{0}\right) \\
& +\frac{1}{2}\left(U_{h}^{n+1}-U_{h}^{n}\right)^{\top} S\left(U_{h}^{n+1}+U_{h}^{n}\right) .
\end{aligned}
$$

Note that from (32a) $\frac{\Delta t}{2}\left(U_{h, t}^{n+1}+U_{h, t}^{n}\right)=U_{h}^{n+1}-U_{h}^{n}+\Delta t\left(\frac{1}{2}-\theta\right)\left(U_{h, t}^{n+1}-U_{h, t}^{n}\right)$, it follows that

$$
\begin{aligned}
& \Delta \mathcal{E}_{h}^{n}=\left(\frac{1}{2}-\theta\right)\left(U_{h}^{n+1}-U_{h}^{n}\right)^{\top} S\left(U_{h}^{n+1}-U_{h}^{n}\right)-\left(U_{h}^{n+1}-U_{h}^{n}\right)^{\top}\left(\left((1-\theta) \lambda^{n}+\theta \lambda^{n+1}\right) e_{0}\right) \\
& +\Delta t\left(\frac{1}{2}-\theta\right)\left(U_{h, t}^{n+1}-U_{h, t}^{n}\right)^{\top}\left((1-\theta)\left(-S U_{h}^{n}-\lambda^{n} e_{0}\right)+\theta\left(-S U_{h}^{n+1}-\lambda^{n+1} e_{0}\right)\right) .
\end{aligned}
$$

Clearly we have

$$
\Delta t\left((1-\theta)\left(-S U_{h}^{n}-\lambda^{n} e_{0}\right)+\theta\left(-S U_{h}^{n+1}-\lambda^{n+1} e_{0}\right)\right)=M\left(U_{h, t}^{n+1}-U_{h, t}^{n}\right),
$$

which allows us to conclude that (33) holds.

Finally we establish the energy evolution associated to (34) in the particular case where $\beta=\frac{1}{4}$. To this aim, let us introduce the energy evolution as follows:

$$
\Delta \mathcal{E}_{h}^{n}=\left[\frac{1}{2} U_{h, t}^{\top} M U_{h, t}+\frac{1}{2} U_{h}^{\top} S U_{h}\right]_{n-\frac{1}{2}}^{n+\frac{1}{2}}
$$


which leads to

$$
\Delta \mathcal{E}_{h}^{n}=\frac{1}{2}\left(U_{h, t}^{n+\frac{1}{2}}-U_{h, t}^{n-\frac{1}{2}}\right)^{\top} M\left(U_{h, t}^{n+\frac{1}{2}}+U_{h, t}^{n-\frac{1}{2}}\right)+\frac{1}{2}\left(U_{h}^{n+\frac{1}{2}}-U_{h}^{n-\frac{1}{2}}\right)^{\top} S\left(U_{h}^{n+\frac{1}{2}}+U_{h}^{n-\frac{1}{2}}\right),
$$

where

$$
U_{h}^{n+\frac{1}{2}} \stackrel{\text { def }}{=} \frac{1}{2}\left(U_{h}^{n}+U_{h}^{n+1}\right) \quad \text { and } \quad U_{h}^{n-\frac{1}{2}} \stackrel{\text { def }}{=} \frac{1}{2}\left(U_{h}^{n}+U_{h}^{n-1}\right) .
$$

Then it comes that

$U_{h}^{n+\frac{1}{2}}+U_{h}^{n-\frac{1}{2}}=\frac{1}{2}\left(U_{h}^{n+1}+2 U_{h}^{n}+U_{h}^{n-1}\right) \quad$ and $\quad U_{h}^{n+\frac{1}{2}}-U_{h}^{n-\frac{1}{2}}=\frac{1}{2}\left(U_{h}^{n+1}-U_{h}^{n-1}\right)$,

and also

$$
U_{h}^{n+1}=U_{h}^{n}+\Delta t U_{h, t}^{n+\frac{1}{2}} \quad \text { and } \quad U_{h}^{n}=U_{h}^{n-1}+\Delta t U_{h, t}^{n-\frac{1}{2}}
$$

so we have

$$
U_{h, t}^{n+\frac{1}{2}}-U_{h, t}^{n-\frac{1}{2}}=\frac{U_{h}^{n+1}-2 U_{h}^{n}+U_{h}^{n-1}}{\Delta t} \quad \text { and } \quad U_{h, t}^{n+\frac{1}{2}}+U_{h, t}^{n-\frac{1}{2}}=\frac{U_{h}^{n+1}-U_{h}^{n-1}}{\Delta t} .
$$

Obviously, we get

$$
\Delta \mathcal{E}_{h}^{n}=\frac{1}{2}\left(U_{h}^{n+1}-U_{h}^{n-1}\right)^{\top} M\left(\frac{U_{h}^{n+1}-2 U_{h}^{n}+U_{h}^{n-1}}{\Delta t^{2}}\right)+S\left(\frac{U_{h}^{n+1}+2 U_{h}^{n}+U_{h}^{n-1}}{4}\right) .
$$

According to (34) together with $\beta=\frac{1}{4}$, we deduce that

$$
\Delta \mathcal{E}_{h}^{n}=\frac{1}{2}\left(U_{h}^{n+1}-U_{h}^{n-1}\right)^{\top}\left(-\lambda^{n+1} e_{0}\right)=\frac{1}{2}\left(u_{0}^{n+1}-u_{0}^{n-1}\right)\left(-\lambda^{n+1}\right) .
$$

Since $u_{0}^{n+1}-u_{0}^{n-1}=(1+e) u_{0}^{n, e}-u_{0}^{n-1}$, we find

$$
\Delta \mathcal{E}_{h}^{n}=\frac{1}{2}(1+e)\left(\left(-\lambda^{n+1} u_{0}^{n, e}\right)-\left(-\lambda^{n+1} u_{0}^{n-1}\right)\right)=\frac{1}{2}(1+e) \lambda^{n+1} u_{0}^{n-1}
$$

\section{References}

[1] H. Brézis, Opérateurs maximaux monotones et semi-groupes de contractions dans les espaces de Hilbert, North-Holland Publishing Co., Amsterdam, 1973. North-Holland Mathematics Studies, No. 5. Notas de Matemática (50). 
[2] H. Brezis, Analyse fonctionnelle, Collection Mathématiques Appliquées pour la Maîtrise. [Collection of Applied Mathematics for the Master's Degree], Masson, Paris, 1983. Théorie et applications. [Theory and applications].

[3] N.J. Carpenter, R.L. Taylor, M.G. Katona, Lagrange constraints for transient finite element surface contact., Int. J. Numer. Meth. Engng. 32 (1991) 103-128.

[4] V. Chawla, T.A. Laursen, Energy consistent algorithms for frictional contact problems, Internat. J. Numer. Methods Engrg. 42 (1998) 799827.

[5] F. Dabaghi, A. Petrov, J. Pousin, Y. Renard, Convergence of mass redistribution method for the one-dimensional wave equation with a unilateral constraint at the boundary, ESAIM Math. Model. Numer. Anal. 48 (2014) 1147-1169.

[6] P. Deuflhard, R. Krause, S. Ertel, A contact-stabilized Newmark method for dynamical contact problems, Internat. J. Numer. Methods Engrg. 73 (2008) 1274-1290.

[7] D. Doyen, A. Ern, S. Piperno, Time-integration schemes for the finite element dynamic Signorini problem, SIAM J. Sci. Comput. 33 (2011) 223-249.

[8] Y. Dumont, L. Paoli, Vibrations of a beam between obstacles. Convergence of a fully discretized approximation, M2AN Math. Model. Numer. Anal. 40 (2006) 705-734.

[9] C. Eck, J. Jarušek, M. Krbec, Unilateral contact problems : variational methods and existence theorems, Monographs and textbooks in pure and applied mathematics, Chapman \& Hall, Boca Raton, London, New York, 2005.

[10] E. Grosu, I. Harari, Stability of semidiscrete formulations for elastodynamics at small time steps, Finite Elem. Anal. Des. 43 (2007) 533-542.

[11] C. Hager, S. Hüeber, B.I. Wohlmuth, A stable energy-conserving approach for frictional contact problems based on quadrature formulas, Internat. J. Numer. Methods Engrg. 73 (2008) 205-225. 
[12] C. Hager, B.I. Wohlmuth, Analysis of a space-time discretization for dynamic elasticity problems based on mass-free surface elements, SIAM J. Numer. Anal. 47 (2009) 1863-1885.

[13] H.M. Hilber, T..J.R. Hughes, R.L. Taylor, Improved numerical dissipation for time integration algorithms in structural dynamics, Earthquake Engineering \& Structural Dynamics 5 (1977) 283-292.

[14] T.J.R. Hughes, The finite element method, Prentice Hall Inc., Englewood Cliffs, NJ, 1987. Linear static and dynamic finite element analysis, with the collaboration of Robert M. Ferencz and Arthur M. Raefsky.

[15] H. Khenous, P. Laborde, Y. Renard, Mass redistribution method for finite element contact problems in elastodynamics, Eur. J. Mech. A Solids 27 (2008) 918-932.

[16] J.U. Kim, A boundary thin obstacle problem for a wave equation, Comm. Partial Differential Equations 14 (1989) 1011-1026.

[17] S. Krenk, Energy conservation in Newmark based time integration algorithms, Comput. Methods Appl. Mech. Engrg. 195 (2006) 6110-6124.

[18] T.A. Laursen, Computational contact and impact mechanics. Fundamentals of modeling interfacial phenomena in nonlinear finite element analysis., Springer-Verlag, Berlin, Heidelberg, New York, (2003).

[19] T.A. Laursen, V. Chawla, Design of energy conserving algorithms for frictionless dynamic contact problems, Internat. J. Numer. Methods Engrg. 40 (1997) 863-886.

[20] T.A. Laursen, G.R. Love, Improved implicit integrators for transient impact problems-geometric admissibility within the conserving framework, Internat. J. Numer. Methods Engrg. 53 (2002) 245-274.

[21] G. Lebeau, M. Schatzman, A wave problem in a half-space with a unilateral constraint at the boundary, J. Differential Equations 53 (1984) 309-361.

[22] J.L. Lions, E. Magenes, Problèmes aux limites non homogènes et applications. Vol. 1, Travaux et Recherches Mathématiques, No. 17, Dunod, Paris, 1968. 
[23] N.M. Newmark, A method of computational for structural dynamics, ASCE, Journal of the Engineering Mechanics Division, Vol. 85 No. EM3, (1959).

[24] L. Paoli, Time discretization of vibro-impact, R. Soc. Lond. Philos. Trans. Ser. A Math. Phys. Eng. Sci. 359 (2001) 2405-2428. Non-smooth mechanics.

[25] L. Paoli, M. Schatzman, A numerical scheme for impact problems. I ,II. The multidimensional case, SIAM J. Numer. Anal. 40 (2002) 734-768.

[26] A. Petrov, M. Schatzman, Mathematical results on existence for viscoelastodynamic problems with unilateral constraints, SIAM J. Math. Anal. 40 (2008/09) 1882-1904.

[27] C. Pozzolini, M. Salaun, Some energy conservative schemes for vibroimpacts of a beam on rigid obstacles, ESAIM Math. Model. Numer. Anal. 45 (2011) 1163-1192.

[28] Y. Renard, The singular dynamic method for constrained second order hyperbolic equations: application to dynamic contact problems, J. Comput. Appl. Math. 234 (2010) 906-923.

[29] Y. Renard, J. Pommier, Getfem++. An Open Source generic C++ library for finite element methods, http://home.gna.org/getfem. (????).

[30] M. Schatzman, A hyperbolic problem of second order with unilateral constraints: The vibrating string with a concave obstacle, Journal of Mathematical Analysis and Applications 73 (1980) 138-191.

[31] A. Tkachuk, B.I. Wohlmuth, M. Bischoff, Hybrid-mixed discretization of elasto-dynamic contact problems using consistent singular mass matrices, Internat. J. Numer. Methods Engrg. 94 (2013) 473-493.

[32] P. Wriggers, Computational contact mechanics, John Wiley \& Sons Ltd., 2002. 